ARTICLE

https://doi.org/10.1038/s41467-018-07189-8

\title{
MacroH2A1 chromatin specification requires its docking domain and acetylation of $\mathrm{H} 2 \mathrm{~B}$ lysine 20
}

Penelope D. Ruiz \& Matthew J. Gamble 1,2

The histone variant macroH2A1 localizes to two functionally distinct chromatin subtypes marked by either $\mathrm{H} 3 \mathrm{~K} 27 \mathrm{me} 3$ or $\mathrm{H} 2 \mathrm{~B}$ acetylations, where it is thought to directly regulate transcription. The recent finding, that macroH2A1 regulates mitochondrial respiration by globally dampening PARP activity, requires the field to re-evaluate which functions of mac$\mathrm{roH} 2 \mathrm{~A} 1$ are due to global effects on cellular metabolism and which are direct effects determined by macroH2A1 chromatin localization. Here, we demonstrate macroH2A1 incorporation into $\mathrm{H} 2 \mathrm{~B}$-acetylated chromatin requires a feature in its histone-fold domain, distinguishing this process from incorporation into H3K27me3-containing chromatin in which multiple features of macroH2A1 are sufficient for targeting. In addition, we identify H2BK2O acetylation as a critical modification required to target macroH2A1 to $\mathrm{H} 2 \mathrm{~B}$-acetylated chromatin. Our findings have allowed us to definitively establish that macroH2A1's regulation of an important transcriptional program, the senescence-associated secretory phenotype (SASP), requires its accurate genomic localization.

\footnotetext{
${ }^{1}$ Department of Molecular Pharmacology, Albert Einstein College of Medicine, Bronx, NY 10461, USA. ${ }^{2}$ Department of Cell Biology, Albert Einstein College of Medicine, Bronx, NY 10461, USA. Correspondence and requests for materials should be addressed to M.J.G. (email: matthew.gamble@einstein.yu.edu)
} 
C hromatin, made of repeating units of nucleosomes, can be locally, structurally and functionally differentiated by a variety of mechanisms including covalent posttranslational modifications (PTMs) of histones, ATP-dependent remodeling and the incorporation of specialized histone variants, to regulate DNA-dependent reactions ${ }^{1}$. Unlike their canonical counterparts, histone variants are incorporated in a replicationindependent manner to perform a particular function predicated on its targeting to the appropriate genomic locations. There are two fundamental requirements for this targeting. (1) The histone variant must harbor a specific feature differentiating it from other histones. (2) A feature at the site of chromatin incorporation must serve as a beacon to recruit the histone variant to the appropriate genomic locations.

MacroH2A-type histone variants, which include macroH2A1.1, macroH2A1.2, and macroH2A2, are composed of a histone-fold domain (HFD), a basic linker region and a $25 \mathrm{kDa}$ carboxylterminal region called a macrodomain ${ }^{2}$. At three-times the size of canonical $\mathrm{H} 2 \mathrm{~A}$, macroH2A-containing nucleosomes organize the same amount of $\mathrm{DNA}^{2}$. The linker and macrodomain emerge from the nucleosome core where they participate in the recruitment of a host of co-factors $^{3-9}$. Generally, macroH2As create large, specialized chromatin environments, hundreds of kilobases long ${ }^{4,10}$. While the overall structure of the three macroH2A isoforms is conserved, an alternative splicing event renders macroH2A1.1 capable of binding the PTM poly(ADP-ribose)(PAR) produced by PAR polymerases (PARPs), which plays key roles in the ability of macroH2A1.1 to regulate gene transcription ${ }^{4,11}$ and DNA damage responses $^{12}$. Two critical glycines (Gly224 and Gly314) are required for PAR binding ${ }^{11}$. The macrodomains of macroH2A1.2 and macroH2A2 lack these critical glycines and contain a three amino acid insertion which collectively disrupts the stabilizing interactions observed between PAR and macroH2A1.1 ${ }^{11,13}$. The relative level of macroH2A1.1 splicing is significantly perturbed across many types of cancer, resulting in reduced macroH2A1.1 expression $^{14-16}$. Re-expression of macroH2A1.1 suppressed cancer cell proliferation ${ }^{14,17,18}$, suppressed anchorage-independent growth and cell invasiveness in breast cancer ${ }^{19}$, and suppressed metastasis of melanoma in mice ${ }^{18}$. MacroH2A1.1 is thus not only a structural component of chromatin, but also a reader of a functionally diverse PTM with roles in transcriptional regulation and DNA damage responses.

First identified as enriched on the inactive $\mathrm{X}$ chromosome (Xi), macroH2As were considered markers of transcriptionally repressed chromatin ${ }^{13,20}$. However, later work demonstrated that macroH2A's roles in the cell are not so simplistic. The majority of macroH2A is found on autosomes as part of facultative heterochromatin marked by trimethylation of histone $\mathrm{H} 3$ on lysine 27 (H3K27me3) or as part of transcriptionally active euchromatin marked by nine histone acetylations (e.g. H2B at K15 and K20; $\mathrm{H} 3$ at $\mathrm{K} 4, \mathrm{~K} 14$ and $\mathrm{K} 18$; $\mathrm{H} 4$ at $\mathrm{K} 91$; and $\mathrm{H} 2 \mathrm{~A}$ at $\mathrm{K} 5$ ) where it can either positively or negatively regulate transcription $4,5,10,21,22$. The physiological functions of macroH2A are an active area of research; it serves as a barrier to stem cell reprogramming ${ }^{23-25}$, as a regulator of gene expression $22,26-28$ during normal growth and cellular senescence ${ }^{29,30}$. MacroH2A1.1 plays a specialized role in transcriptional regulation; through interaction with $\mathrm{PAR}$, macroH2A1.1 recruits PARP1 and CBP, leading to the acetylation of H2B K12 (H2BK12ac) and K120 (H2BK120ac) ${ }^{4,29}$. This mechanism plays a critical role in oncogene-induced senescence (OIS), an important tumor suppressive mechanism. In OIS, macroH2A1 serves as a component of the senescence-associated heterochromatic foci involved in transcriptional repression ${ }^{30}$. However, macroH2A1.1 is specifically required for the transcriptional activation of a myriad of cytokines, chemokines and metalloproteases that make up the senescence-associated secretory phenotype (SASP), which enables the clearance of senescent cells ${ }^{31}$.

Recently, a global role for macroH2A1.1 in the regulation of mitochondrial function and energy metabolism has been identified. Through its interaction with PAR and concomitant inhibition of PARP1 activity, macroH2A1.1 allows for greater production of $\mathrm{NAD}^{+}$in mitochondria ${ }^{32}$ suggesting that macroH2A1 plays a global role in cellular energetics, regardless of where macroH $2 \mathrm{~A} 1$ localizes on the genome. This result requires the field to carefully re-evaluate which functions of macroH2A1 are mediated by global effects of macroH2A1 on cellular energetics and which require it to function locally through deposition at specific chromatin loci.

Here, we demonstrate that the requirements for deposition of macroH2A 1 into $\mathrm{H} 2 \mathrm{Bac}$ and $\mathrm{H} 3 \mathrm{~K} 27$ me3-marked chromatin are distinct. We find that the carboxyl-terminal region of the HFD of macroH2A1 is required for association with $\mathrm{H} 2 \mathrm{~B}$-acetylated chromatin, whereas multiple features of macroH2A1 are sufficient for association with chromatin marked by H3K27me3. Furthermore, we determined that regulation of macroH2A1dependent transcriptional programs, such as the SASP, requires its ability to accurately localize to its $\mathrm{H} 2 \mathrm{~B}$-acetylated chromatin niche. Additionally, we identified H2BK20ac as a key PTM which serves as a beacon to target macroH2A1 into the $\mathrm{H} 2 \mathrm{~B}$-acetylated chromatin environment. The loss of macroH2A1 from H2Bacetylated chromatin, caused either by mutating macroH2A1 itself or by ablating the $\mathrm{H} 2 \mathrm{BK} 20 \mathrm{ac}$ chromatin cue for macroH2A1 deposition, led to a defect in the upregulation of SASP transcription. Overall, our work highlights key elements regulating the deposition of macroH2A1 into $\mathrm{H} 2 \mathrm{~B}$-acetylated chromatin and demonstrates that macroH2A1 plays critical local roles in regulating gene expression.

\section{Results}

Ectopic wild-type macroH2A1 incorporates into nucleosomes. While several groups, including ours, have determined where macroH2A1 localizes across the genome in several cell types ${ }^{4,25,33-35}$, we know little about the rules governing specification of macroH2A1-containing chromatin. Previous studies have demonstrated that multiple features of macroH2A1 were sufficient to target the histone variant to the predominantly $\mathrm{H} 3 \mathrm{~K} 27 \mathrm{me} 3$-containing $\mathrm{Xi}$, including regions in macroH2A1's HFD and macrodomain ${ }^{36,37}$. Subsequently, we determined that macroH2A1 localized to $\mathrm{H} 3 \mathrm{~K} 27$ me3-containing chromatin across autosomes as well ${ }^{10}$. Additionally, we determined that a similar proportion of macroH2A1 localizes to transcriptionally active chromatin (Fig. 1a) ${ }^{4}$.

These additional findings led us to re-evaluate the rules that dictate macroH2A1 genomic localization. Using our expanded knowledge of macroH2A1 localization and a series of macroH2A1 domain deletion and substitution mutants, we sought to identify the features of macroH2A1 required to specify its accurate deposition into its two chromatin subtypes, H3K27me3-marked chromatin or H2Bac-marked chromatin. To this end, we derived a panel of cell lines from immortalized IMR90 human diploid lung fibroblasts that expressed a mutant macroH2A1 protein harboring a carboxyl-terminal Flag-tag (macroH2A1-Flag). Ectopic expression of macroH2A1.1 has been shown to induce cellular senescence ${ }^{29}$, so ectopically expressed full-length macroH2A1 constructs possessed the macroH2A1.2 macrodomain to establish stable, replicative cell lines. Two lines harbored truncation mutants lacking the macrodomain alone (macroH2A1 $1^{1-160}$-Flag) or lacking both the linker and macrodomain (macroH2A1 $1-122$-Flag) (Fig. 1b). In an additional macroH2A1 mutant the HFD of macroH2A1.2 was replaced 
a

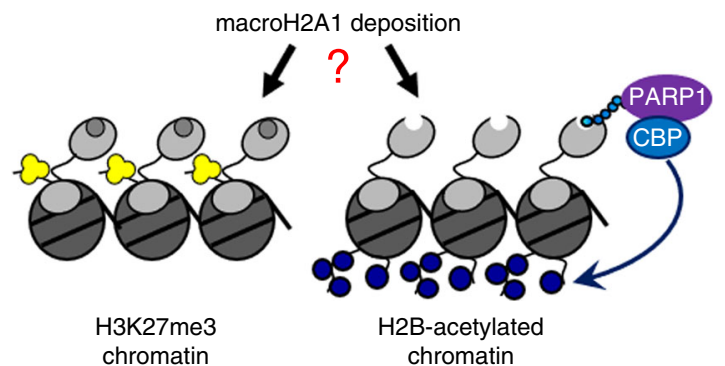

d

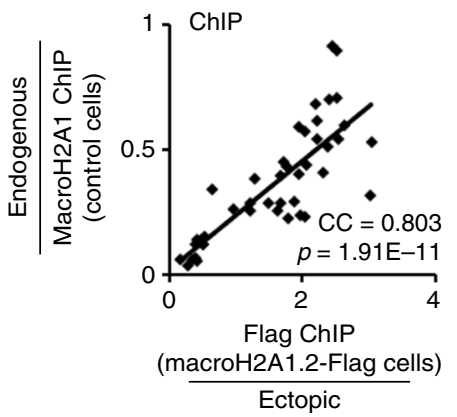

b

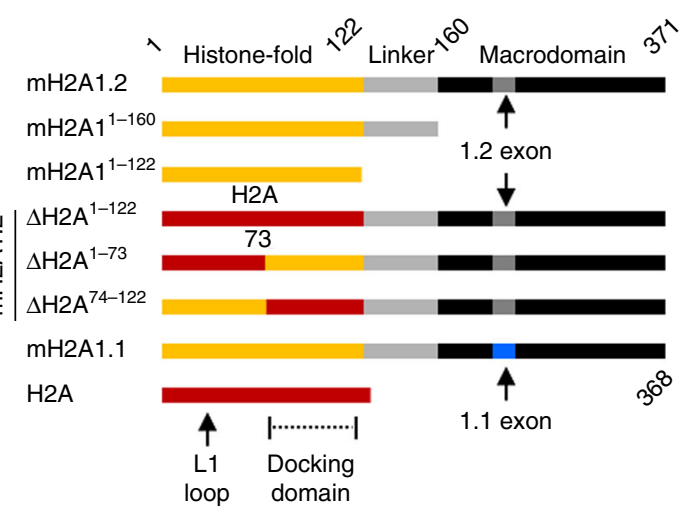

C

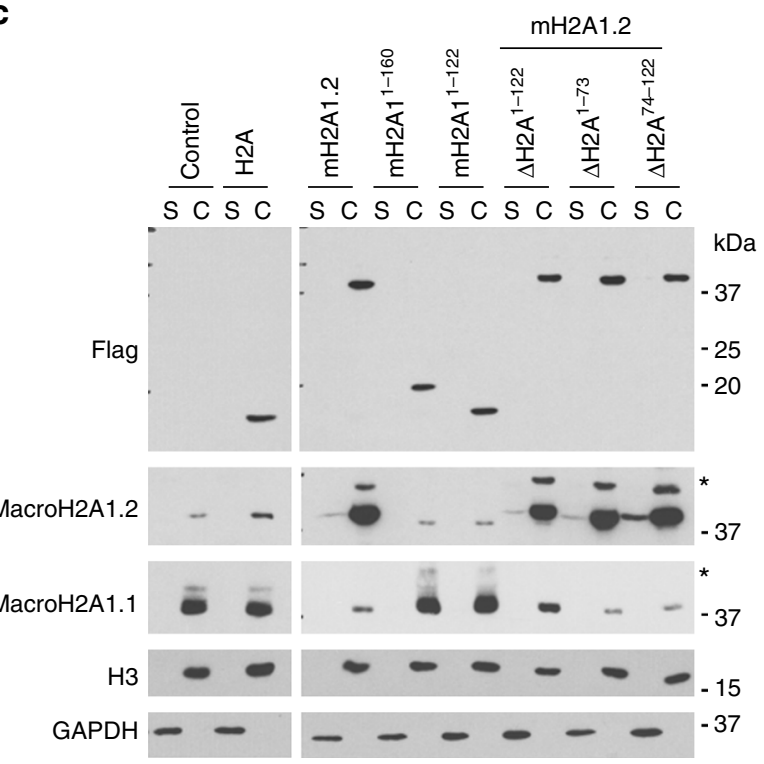

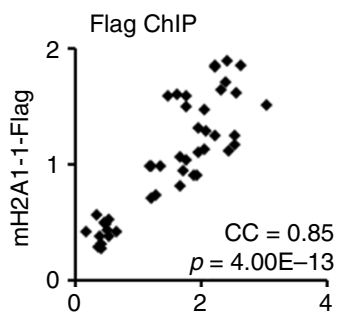

$\mathrm{mH} 2 \mathrm{~A} 1.2-\mathrm{Flag}$

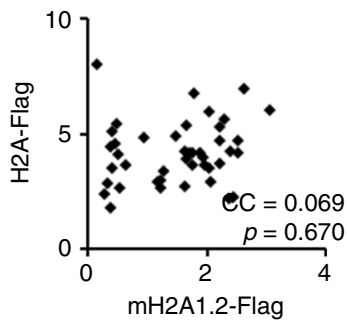

$\mathbf{f}$

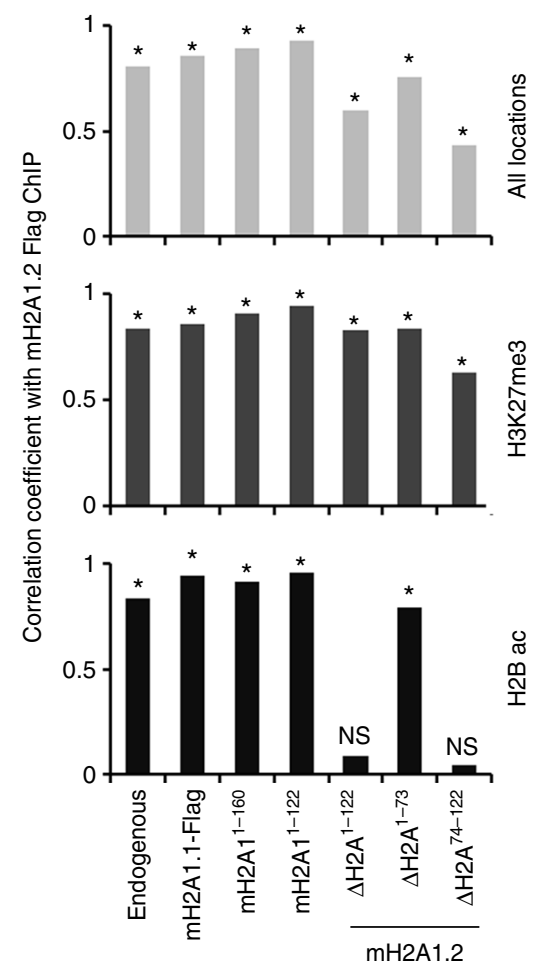

Fig. 1 MacroH2A1 requires the carboxyl-terminal half of its HFD for accurate deposition into H2B-acetylated chromatin. a Schematic depicting the different chromatin environments into which macroH2A1 is deposited marked by either H3K27me3 or acetylations at H2B K12, K15, K20, and K120. In H2Bacetylated chromatin, macroH2A1.1 cooperates with PARP1 through binding PAR to recruit CBP and regulate transcription. $\mathbf{b}$ Diagram of mutant macroH2A1.2-Flag (mH2A1) constructs expressed in IMR90-hTERT cells. The macroH2A1 alternative exons a nd docking domain are indicated. c Immunoblots of soluble (S) and chromatin (C) fractions from IMR90-hTERT cells described in $\mathbf{b}$ for indicated proteins. The minor band $\left(^{\star}\right)$ above the major band in the macroH2A1.1 and macroH2A1.2 immunoblots is the mono-ubiquitylated species of macroH2A1. d Scatter plot of Flag ChIP from wild-type macroH2A1.2-Flag cells plotted against macroH2A1 ChIP from control cells expressing GFP ( $n=3$ independent cell passages). Pearson's correlation coefficient (CC) and associated p-value indicated are from Pearson's product-moment correlation. e Scatter plot of Flag ChIP from wild-type macroH2A1.2Flag cells versus Flag ChIP from H2A-Flag cells. ( $n=3$ independent cell passages). f Pearson's correlation coefficient calculated for Flag ChIP from wildtype macroH2A1.2-Flag cells versus vs. Flag ChIP from cells expressing macroH2A1.2-Flag truncations and domain swap mutants described in $\mathbf{b}$. Top graph contains primers across both types or chromatin. Middle graph contains primers for regions marked with H3K27me3. Bottom graph contains primers for regions marked with H2Bac. ( $n=3$ independent cell passages, ${ }^{\star} p<0.003$ from Pearson's product-moment correlation, NS not significant) 
with the homologous region of canonical H2A (macroH2A1.2 ${ }^{\triangle \mathrm{H} 2 \mathrm{~A} 1-122}$-Flag). To ensure that each ectopic macroH2A1 mutant and wild-type protein could incorporate into chromatin, we fractionated the cells into soluble and chromatin fractions and liberated the mononucleosomes from the chromatin fraction with Micrococcal nuclease (MNase) (Fig. 1c). A Flag immunoblot indicated that the overwhelming majority of the ectopically expressed macroH2A1.2-Flag proteins were chromatin incorporated, similar to endogenous macroH2A1 and $\mathrm{H} 3$.

We previously described a compensatory post-translational feedback mechanism which decreases the level of endogenous macroH2A1 when macroH2A1 is ectopically expressed ${ }^{4}$. The feedback and reduction of macroH2A1.1 can be clearly observed in all the ectopic macroH2A1.2-Flag mutant lines that harbor an intact macrodomain (Fig. 1c); however, the macroH2A1-Flag lines lacking the macrodomain (e.g., macroH $2 \mathrm{Al}^{1-160}$ and macroH2A $1^{1-122}$ ) failed to reduce endogenous macroH2A1.1 indicating that the macrodomain plays a critical role in this compensatory feedback loop.

Multiple features of macroH2A1 target it to H3K27me3. Having ensured that ectopic macroH2A1-Flag proteins incorporate into chromatin and do not significantly alter nuclear size (Supplementary Fig. 1a-b), we turned our attention to determining where they were being deposited using Flag chromatin immunoprecipitation assayed by quantitative PCR (ChIP-qPCR). Using our previous ChIP-seq data ${ }^{4}$ and publicly available data ${ }^{38}$, we designed ChIP primers targeting regions with and without macroH2A1 enrichment in both H2Bac and H3K27me3containing chromatin (Supplementary Table 1). Data from endogenous macroH2A1 ChIP in parental cells highly correlated to data from a Flag ChIP from cells ectopically expressing wildtype macroH2A1.2-Flag, demonstrating that ectopically expressed wild-type macroH2A1.2 was genomically targeted in a manner similar to the endogenous protein (Fig. 1d, Supplementary Fig. 2). The correlation remained high when looking specifically at association with either $\mathrm{H} 2 \mathrm{Bac}$ chromatin or $\mathrm{H} 3 \mathrm{~K} 27$ me3-marked chromatin indicating that ectopic wild-type macroH2A1.2 correctly localizes to both chromatin environments (Fig. 1f). Consistent with a distinct pattern of deposition, there was no significant correlation between the Flag ChIPs from cells expressing macroH2A1.2-Flag and canonical H2A-Flag (Fig. 1e, Supplementary Fig. 2).

Armed with the Flag ChIP-qPCR approach, we sought to determine which macroH2A1 domains are required to correctly specify macroH2A1-containing chromatin. When we compared the Flag ChIP data from wild-type macroH2A1.2-Flag expressing cells with data from cells expressing macroH2A $1^{1-160}$-Flag, lacking the macrodomain, or macroH2A1 ${ }^{1-122}$-Flag, lacking the linker region and macrodomain, we found that either mutant demonstrated a high correlation with wild-type macroH2A1.2Flag, indicating that neither of those domains are required for the specification of macroH2A1-containing chromatin and that the HFD of macroH2A1 is sufficient to drive this process (Fig. 1f).

To test the role of the HFD in the specification of macroH2A1containing H3K27me3 chromatin, we performed Flag ChIP on cells expressing macroH2A1. $2^{\Delta \mathrm{H} 2 \mathrm{~A} 1-122}$-Flag. This mutant correlated well with wild-type macroH2A1.2-Flag ChIP, indicating that the HFD of macroH2A1.2 is also not required for targeting its deposition into H3K27me3-marked chromatin (Fig. If, Supplementary Fig. 3). The fact that all three domain mutants could localize to $\mathrm{H} 3 \mathrm{~K} 27 \mathrm{me} 3$-marked chromatin in a manner similar to wild-type indicates that multiple features of macroH2A1 can target its deposition to H3K27me3-marked chromatin. Overall, this is consistent with previous studies on the localization of macroH2A1 to the H3K27me3-marked $\mathrm{Xi}^{36,37}$.

A previous report showed that replacing the L1 loop and $\alpha 1$ helix of canonical $\mathrm{H} 2 \mathrm{~A}$ with the homologous regions of macroH2Al in the absence of the macroH2Al linker and macrodomain was sufficient for enrichment on the inactive $\mathrm{X}^{36}$. We expressed a chimera containing the $\mathrm{N}$-terminal half of macroH2A1, possessing the macroH2A1 L1 loop and al helix, fused to the C-terminal half of canonical H2A (macroH2A1-

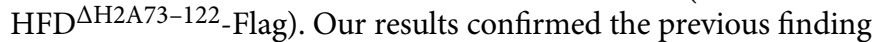
showing that this chimera was accurately deposited into autosomal H3K27me3-containing chromatin (Supplementary Fig. 3). Together our results extend earlier findings by demonstrating that multiple regions of macroH2A1 are sufficient to target this histone variant to H3K27me3-marked autosomal heterochromatin in addition to the previously described H3K27me3-marked Xi.

Critical role of the HFD in specifying macroH2A1 localization. Previously, our lab demonstrated that most macroH2A1regulated genes in IMR90 cells are found in chromatin cooccupied by macroH2A1 and H2Bac, making this type of chromatin particularly important for macroH2A1's functions ${ }^{4,29}$. MacroH2A1 mutants lacking the macrodomain or the macrodomain and linker domain highly correlate with wild-type macroH2A1.2 association in H2Bac chromatin (Fig. 1f). Accordingly, neither domain is required for macroH2Al association with $\mathrm{H} 2 \mathrm{Bac}$ chromatin. However, the macroH2A1.2 ${ }^{\Delta \mathrm{H} 2 \mathrm{Al} 1-122}$-Flag mutant, where the HFD was replaced with canonical H2A, does not show a significant correlation with wild-type macroH2A1.2Flag in H2Bac chromatin (Fig. 1f). This indicates a specific requirement for the HFD in targeting macroH2A1 to $\mathrm{H} 2 \mathrm{Bac}$ chromatin, differentiating it from the rules governing macroH2A1 association with H3K27me3-marked chromatin.

The HFD of macroH2A1 is more highly conserved across vertebrates than canonical $\mathrm{H}_{2} \mathrm{~A}^{39}$. Amino acid sequence alignment between H2A-type histones indicate regions of difference between macroH2A and the other H2A-type histones that may confer specificity to macroH2A chromatin deposition (Supplementary Fig. 4). Two key regions of divergence are the L1-loop and the docking domain. The L1-loop creates the only interface between the two H2A-H2B dimers within a single nucleosome. The structurally distinct L1-loop of macroH2A1 regulates the preference of macroH2A1 to form heterotypic nucleosomes with canonical $\mathrm{H}_{2} \mathrm{~A}^{2,40}$. The $\mathrm{H} 2 \mathrm{~A}$ docking domain, composed of two short helices and containing a conserved acidic patch, interacts with the $\mathrm{H} 3 / \mathrm{H} 4$ tetramer and locks the $\mathrm{H} 3 \quad \alpha-\mathrm{N}$ helix in position $^{41}$. MacroH2A1 and canonical H2A docking domains harbor several amino acid differences, but demonstrate high structural similarity ${ }^{2}$. The docking domain of H2A.Z, specifically the acidic patch, was shown to be required for H2A.Z deposition and its function ${ }^{42,43}$.

To determine which feature was dominant in specifying macroH2A1-containing-H2Bac chromatin, we used a chimerabased approach similar to one used to identify regions of H2A.Z critical for its correct localization ${ }^{43,44}$. Each half of the HFD of macroH2A1.2 was replaced with the homologous region of canonical H2A (Fig. 1b). Replacement of the amino-terminal half of the HFD of macroH2A1 with canonical H2A (macroH2A1.2 ${ }^{\Delta \mathrm{H} 2 \mathrm{~A} 1-73}$-Flag) did not disrupt specification of macroH2A1-containing chromatin. However, replacement of the carboxyl-terminal half of the HFD of macroH2A1 with the homologous region of canonical H2A (macroH2A1.2 ${ }^{\Delta \mathrm{H} 2 \mathrm{~A} 74-122}$ Flag) failed to significantly correlate with wild-type macroH2A1.2, specifically in the H2Bac-marked chromatin 
a

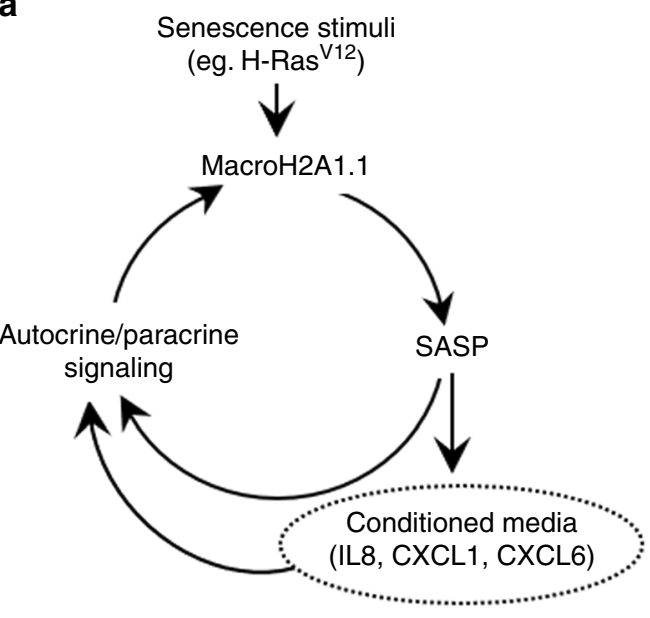

C

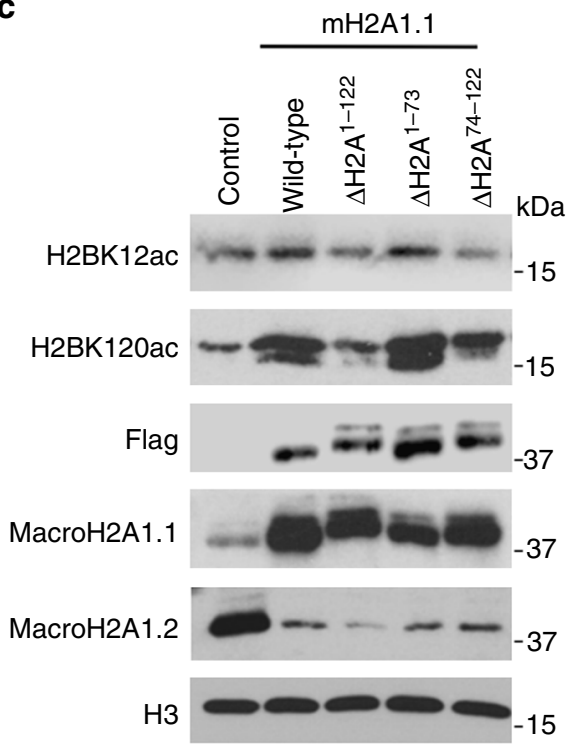

b

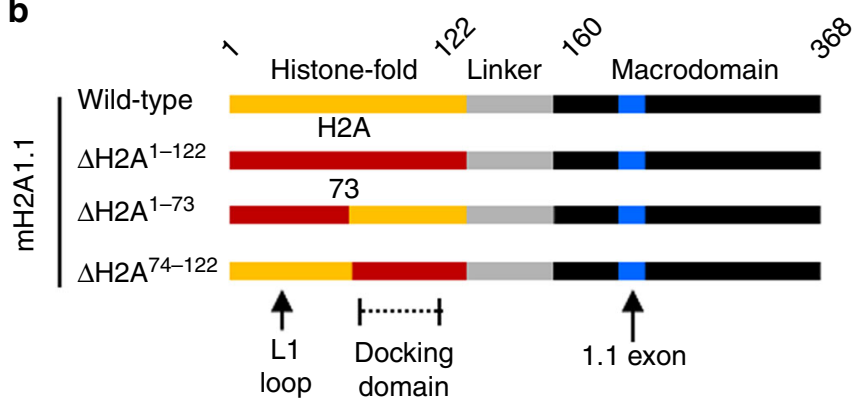

d

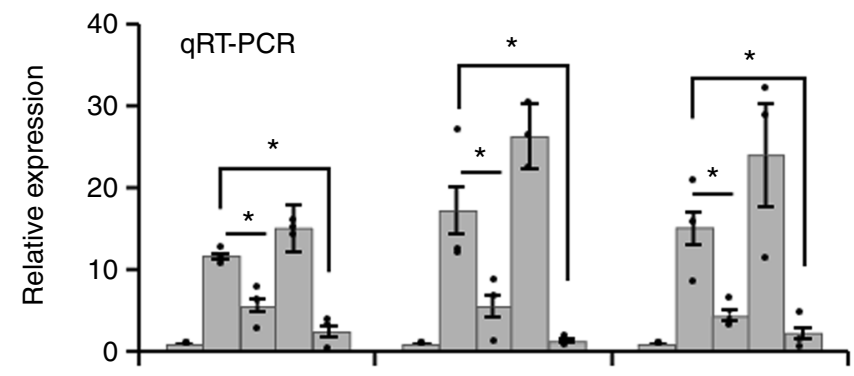

e

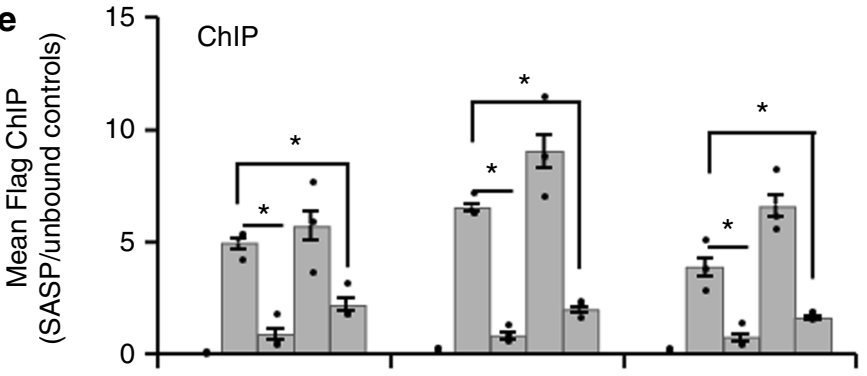

Control
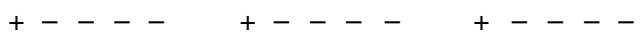

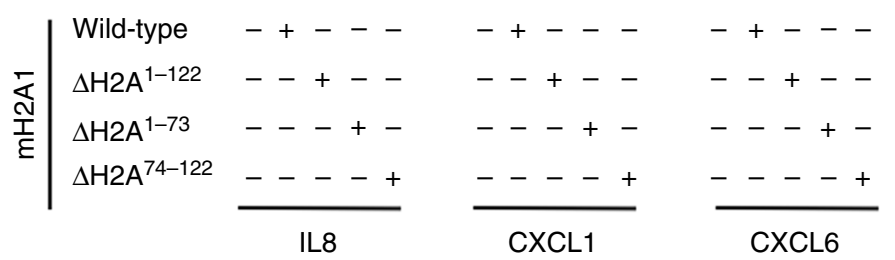

Fig. 2 MacroH2A1 localization to H2Bac chromatin is required for regulation of H2BK12ac, H2BK12Oac, and SASP gene transcription. a Diagram illustrates macroH2A1.1-dependent positive feedback loop regulating the induction of SASP gene expression. Senescent stimuli, such as $\mathrm{H}$-Ras $\mathrm{V} 12$ upregulates the expression of macroH2A1.1 which promotes the transcription of SASP genes. Secreted SASP factors can act in an autocrine or paracrine fashion. Conditioned media from senescent cells contains secreted cytokines and chemokines (SASP factors) which can promote senescence through paracrine signaling. b Schematic of domain swap macroH2A1.1-Flag (mH2A) mutant lentiviral constructs expressed in IMR90-hTERT cells. MacroH2A1.1 exon and docking domain indicated. $\mathbf{c}$ Immunoblots of acid-extracted histones for indicated histones and histone PTMs from cells that ectopically express macroH2A1.1 constructs described in $\mathbf{b}$. d RT-qPCR for SASP factors of cells described in $\mathbf{b}$. Error bars, s.e.m. ( $n=3$ independent lentiviral infections, ${ }^{\star} p<0.05$ from a twotailed Student's $t$-test). e Flag ChIP-qPCR in macroH2A1.2-Flag cells for SASP genes shown in d. Bars represent enrichment of macroH2A1-Flag at SASP loci over unbound control regions. Error bars, s.e.m. ( $n=3$ independent cell passages, ${ }^{\star} p<0.05$ from a two-tailed Student's $t$-test)

(Fig. 1f) as did a similar chimera lacking the macrodomain (Supplementary Fig. 2). Overall, this indicates that the carboxylterminal half of macroH2A1's HFD is required for accurate deposition of this histone variant into its $\mathrm{H} 2 \mathrm{Bac}$ chromatin environment.

MacroH2A1 localization regulates transcriptional program. Cellular senescence, triggered by telomere dysfunction, oncogenic stress, or DNA damage, results in stable withdrawal from the cell cycle through the engagement of tumor suppressor pathways ${ }^{31}$. An additional feature of senescence is the SASP, a transcriptional program inducing the expression of a host of secreted inflammatory factors that contribute to recruitment of innate immune effectors and reinforce the senescent state through paracrine signaling (Fig. $2 \mathrm{a})^{31}$. SASP genes reside in macroH2A1containing, H2Bac-marked chromatin and require macroH2A1.1-mediated recruitment of PARP1 and the acetyltransferase $\mathrm{CBP}$ for their activation ${ }^{29}$. Our results strongly suggested that macroH2A1.1 plays a local and direct role in the transcriptional activation of SASP genes. However, recently it was shown that macroH2A1.1, through its ability to inhibit PARP1, also modulates the level of $\mathrm{NAD}^{+}$available for mitochondrial respiration and global energy metabolism ${ }^{32}$. These findings lead to an important question: Is the regulation of transcription by 
macroH2A1.1 a direct effect of a local association and function of macroH2A 1.1 at the genes it is transcriptionally regulating or is it a downstream consequence of macroH2A1.1's ability to regulate cellular metabolism?

To answer this question, we generated three mutants described above in the context of macroH2A1.1-Flag (e.g., macroH2A $1.1^{\Delta \mathrm{H} 2 \mathrm{~A} 1-122}$, macroH2A1.1 $\left.{ }^{\Delta \mathrm{H} 2 \mathrm{~A} 1-73}, \mathrm{macroH}^{\mathrm{A}} 1.1^{\Delta \mathrm{H} 2 \mathrm{~A} 74-122}\right)$ with the knowledge that replacement of the entire HFD or the carboxyl-terminal half of the HFD leads to aberrant macroH2A1 localization (Fig. 2b). Importantly, there is a strong correlation between the deposition of ectopically expressed macroH2A1.1_Flag and ectopically expressed macroH2A1.2_Flag (Fig. 1e, f, Supplementary Fig. 2) indicating the two macroH2A1 isoforms occupy similar regions in the genome. Immunoblotting for Flag and macroH2A1.1 indicated that the ectopic macroH2A1.1 proteins were similarly expressed (Fig. 2c) and chromatin incorporated (Supplementary Fig. 5). As we have shown previously ${ }^{29}$, ectopic expression of wildtype macroH2A1.1-Flag induced SASP gene expression as assayed by monitoring three SASP genes (e.g., IL8, CXCL1, and CXCL6) by RTqPCR. The results demonstrated that localization deficient macroH2A1.1-Flag mutants, macroH2A1.1 ${ }^{\Delta \mathrm{H} 2 \mathrm{~A} 1-122}$ and macroH2A1.1 ${ }^{\Delta \mathrm{H} 2 \mathrm{~A} 74-122}$, were defective in their ability to induce SASP gene expression (Fig. 2d). As a control, we confirmed the ability of macroH2A1.1 $1^{\triangle \mathrm{H} 2 \mathrm{~A} 1-73}$ to correctly localize to the SASP genes by performing Flag ChIP (Fig. 2e). Overall, these results highlight the importance of the local function of macroH2A1, and demonstrate that macroH2A1.1 localization to its target genes is critical to regulate their transcription.

MacroH2A1.1 chromatin targeting regulates $\mathrm{H} 2 \mathrm{BK} 12 \mathrm{ac}$ and K120ac. We previously demonstrated that macroH2A1.1, in conjunction with PARP1, recruits the acetyltransferase CBP to promote $\mathrm{H} 2 \mathrm{BK} 12 \mathrm{ac}$ and $\mathrm{H} 2 \mathrm{BK} 120 \mathrm{ac}^{4}$. When either wild-type macroH2A 1.1 or macroH2A1.1 ${ }^{\Delta \mathrm{H} 2 \mathrm{~A} 1-73}$, which can correctly localize to chromatin, is ectopically expressed, this regulation manifests as an increase in total H2BK12ac and H2BK120ac levels (Fig. 2c). The effect of macroH2A1.1 on these PTMs is abrogated in the macroH2A1.1 localization defective mutants, macroH2A $1.1^{\triangle \mathrm{H} 2 \mathrm{~A} 1-122}$ and macroH2A1.1 $1^{\Delta \mathrm{H} 2 \mathrm{~A} 74-122}$ (Fig. 2c). Together these data indicate that macroH2A1.1 must be localized to its appropriate chromatin niche to promote H2BK12ac and H2BK120ac. We hypothesized there must be additional, yet unidentified, factors at the endogenous chromatin niche that influence the ability of macroH2A1.1 to regulate the CBPmediated acetylation of $\mathrm{H} 2 \mathrm{~B}$ at $\mathrm{K} 12$ and $\mathrm{K} 120$, and subsequently macroH2A1-dependent gene transcription.

Defining the macroH2A1-containing H2Bac chromatin niche. We next sought to uncover the additional features of the macroH2A1-containing $\mathrm{H} 2 \mathrm{Bac}$ chromatin niche required for macroH2A1.1 to promote H2BK12ac and H2BK120ac and thereby regulate target gene transcription. Of the nine acetylations enriched in macroH2A1-containing chromatin ${ }^{4}$, only two of those acetylations, H2BK15ac and H2BK20ac, exhibit a stronger genome-wide enrichment for macroH2A1 than the acetylations regulated by macroH2A1.1 (e.g., H2BK12ac and H2BK120ac) (Fig. 3a), leading us to hypothesize that H2BK15ac and/or $\mathrm{H} 2 \mathrm{BK} 20 \mathrm{ac}$ play a critical role in macroH2A1's local chromatin environment, impacting its function.

To evaluate the role of H2BK15ac and H2BK20ac in the macroH2A1 chromatin niche, we ablated these marks in IMR90 cells. Depleting H2BK15ac and H2BK20ac through enzyme inhibition, shRNA or CRISPR/Cas9 strategies is impractical as multiple promiscuous enzymes are capable of acetylating these sites $^{45-47}$. Therefore, we decided to replace endogenous H2B with ectopically expressed $\mathrm{H} 2 \mathrm{~B}$ harboring lysine-to-arginine mutations at position 15 or 20 to render these sites incapable of acetylation. To replace endogenous $\mathrm{H} 2 \mathrm{~B}$, a highly expressed protein, we generated stable cells simultaneously expressing two $\mathrm{H} 2 \mathrm{~B}$ lentiviral constructs with identical mutations (e.g., K15R or K20R) and either a carboxyl-terminal Flag or HA tag (Fig. 3b). A Coomassie-stained gel of total histones extracted from these cells shows that the wild-type and mutant $\mathrm{H} 2 \mathrm{~B}$ constructs replaced the overwhelming majority of endogenous H2B (Fig. 3c). We further validated the reduction of endogenous $\mathrm{H} 2 \mathrm{~B}$ using an antibody which recognizes endogenous $\mathrm{H} 2 \mathrm{~B}$ but does not react with $\mathrm{H} 2 \mathrm{~B}$ harboring a carboxy-terminal tag (Fig. 3d). As expected, cells expressing the $\mathrm{H} 2 \mathrm{~B}^{\mathrm{K} 15 \mathrm{R}}$ showed a loss of $\mathrm{H} 2 \mathrm{BK} 15 \mathrm{ac}$, while cells expressing $\mathrm{H} 2 \mathrm{~B}^{\mathrm{K} 20 \mathrm{R}}$ showed a loss of $\mathrm{H} 2 \mathrm{BK} 20 \mathrm{ac}$ (Fig. $3 \mathrm{~d}$ ). Importantly, there was no observable crosstalk between these two marks, as loss of H2BK15ac did not affect the level of H2BK20ac and vice versa. Furthermore, cells harboring either the $\mathrm{H} 2 \mathrm{~B}^{\mathrm{K} 15 \mathrm{R}}$ or $\mathrm{H} 2 \mathrm{~B}^{\mathrm{K} 20 \mathrm{R}}$ mutants did not show altered levels of macroH2A1.1 or macroH2A1.2 (Fig. 3d).

With controls in place, we examined the effect of the $\mathrm{H} 2 \mathrm{~B}$ mutants on H2BK12ac and H2BK120ac. While there was no effect on either mark in cells expressing ectopic $\mathrm{H} 2 \mathrm{~B}^{\mathrm{K} 15 \mathrm{R}}$, there was a striking reduction in both H2BK12ac and H2BK120ac in cells expressing the $\mathrm{H} 2 \mathrm{~B}^{\mathrm{K} 20 \mathrm{R}}$ mutant histone (Fig. 3d). Overall, this indicates that $\mathrm{H} 2 \mathrm{BK} 20 \mathrm{ac}$ is required for the acetylation of $\mathrm{H} 2 \mathrm{~B}$ at $\mathrm{K} 12$ and $\mathrm{K} 120$ and implicates this histone modification in the broader function of macroH2A1.

H2BK20ac promotes the accurate localization of macroH2A1. We hypothesized that H2BK20ac functions as a beacon to specify macroH2A1 localization to its $\mathrm{H} 2 \mathrm{~B}$-acetylated chromatin environment. To test this hypothesis, we liberated mononucleosomes from chromatin of ectopic wild-type $\mathrm{H} 2 \mathrm{~B}, \mathrm{H} 2 \mathrm{~B}^{\mathrm{K} 15 \mathrm{R}}$ and $\mathrm{H} 2 \mathrm{~B}^{\mathrm{K} 20 \mathrm{R}}$ expressing cells using MNase (Fig. 4a). As we were most interested in the association of macroH2A1 with euchromatic, H2Bacetylated chromatin, we employed a mononucleosome preparation protocol which primarily yields euchromatic mononucleosomes ${ }^{48}$. The euchromatin nature of the liberated mononucleosomes was verified by the presence of euchromatic markers, such as H3K4me3 and H3K27ac, and the absence of the heterochromatic H3K27me3 (Fig. 4b). We used Flag antibody to immunoprecipitate only mononucleosomes containing the ectopic wild-type and mutant $\mathrm{H} 2 \mathrm{Bs}$ (Fig. 4c). Cells harboring the $\mathrm{H} 2 \mathrm{~B}^{\mathrm{K} 15 \mathrm{R}}$ mutant consistently demonstrated increased levels of macroH2A1.1 liberation upon MNase digestion (Fig. 4b) and nucleosomes harboring ectopic wild-type $\mathrm{H} 2 \mathrm{~B}$ or $\mathrm{H} 2 \mathrm{~B}^{\mathrm{K} 15 \mathrm{R}}$ demonstrated a robust enrichment of macroH2A1.1. However, mononucleosomes containing $\mathrm{H} 2 \mathrm{~B}^{\mathrm{K} 20 \mathrm{R}}$ failed to incorporate macroH2A1.1 (Fig. 4c), suggesting a critical role for H2BK20ac in mediating macroH2A1 incorporation.

To confirm and extend these results, we measured the relative amount of macroH2A1 localizing to both its $\mathrm{H} 2 \mathrm{Bac}$ and H3K27me3 subtypes using endogenous macroH2A1 ChIP in the ectopic H2B expressing cell lines. Consistent with the results described above, macroH2A1 association with $\mathrm{H} 2 \mathrm{~B}$-acetylated euchromatin sites was significantly reduced in cells expressing $\mathrm{H} 2 \mathrm{~B}^{\mathrm{K} 20 \mathrm{R}}$ compared to wild-type H2B (Fig. 5a). However, macroH2A1 association with H2B-acetylated chromatin sites were unaltered in cells expressing ectopic $\mathrm{H} 2 \mathrm{~B}^{\mathrm{K} 15 \mathrm{R}}$. No significant difference was seen in the levels of endogenous macroH2A1 associated with H3K27me3-marked, macroH2A1-containing chromatin or in regions typically devoid of macroH2A1 (Fig. 5a). Overall, these results demonstrate that $\mathrm{H} 2 \mathrm{BK} 20 \mathrm{ac}$ is critical for targeting macroH2A1 to its $\mathrm{H} 2 \mathrm{~B}$-acetylated, euchromatic chromatin subtype. 


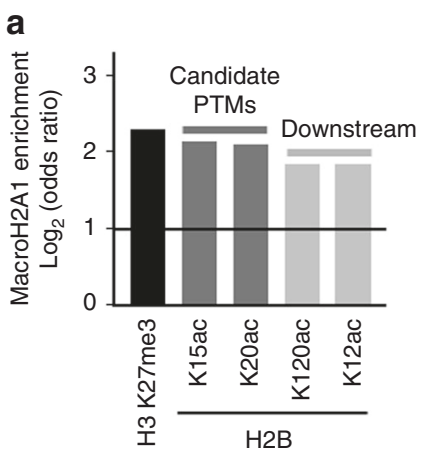

b

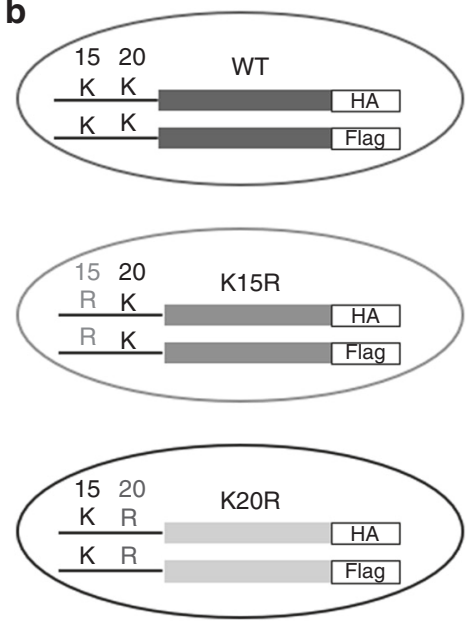

C

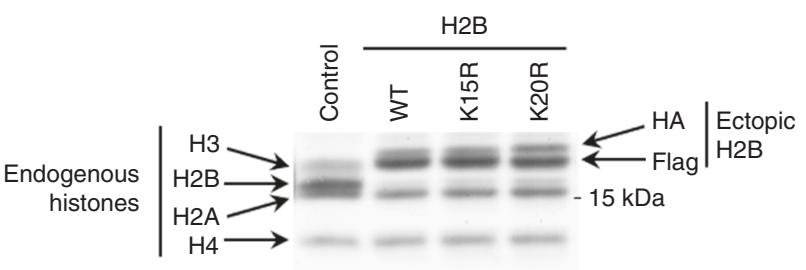

d

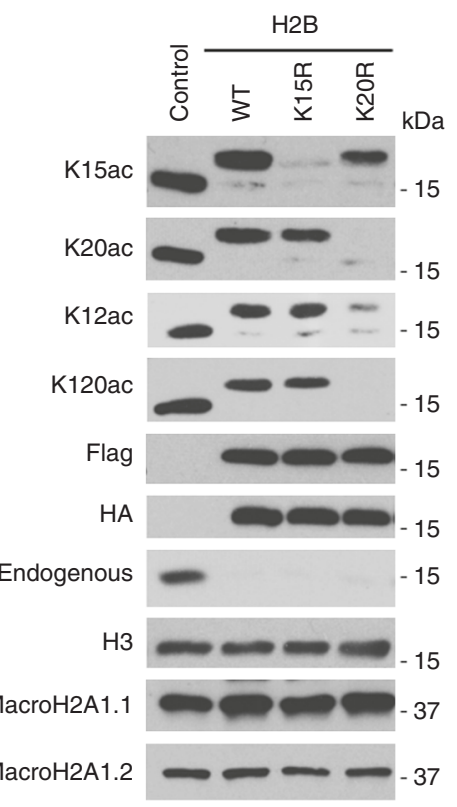

Fig. $3 \mathrm{H} 2 \mathrm{BK} 2 \mathrm{Oac}$ is required for acetylation of $\mathrm{H} 2 \mathrm{~B}$ on $\mathrm{K} 12$ and $\mathrm{K} 120$. a ChIP-seq data for macroH2A1 and 5 histone PTMs ${ }^{4}$ enriched for macroH2A1 in IMR90 cells. b Schematic for co-expression of two ectopic H2B constructs in IMR90-hTERT cells to ablate H2BK15ac and H2BK20ac. (WT, wild-type). c Coomassie-stained gel of acid-extracted histones from cells described in $\mathbf{b}$. Bands corresponding to histones are indicated. $\mathbf{d}$ Immunoblots of acidextracted histones from cell lines described in $\mathbf{b}$ for indicated H2B PTMs and proteins

$\mathrm{H} 2 \mathrm{BK} 20 \mathrm{ac}$ is required for macroH2A1-regulated transcription. Earlier we demonstrated, using macroH2A1 mutants, that correct localization of macroH2A1 is critical for the transcriptional regulation of SASP genes (Fig. 2d). Armed with an understanding of the role of H2BK20ac in macroH2A1 localization, we sought to confirm this result with an independent approach. SASP gene expression can be robustly triggered through paracrine signaling whereby cytokines produced by senescent cells initiate SASP activation in neighboring cells ${ }^{31,49,50}$. We applied conditioned media from cells induced to senescence by oncogenic Ras ${ }^{\mathrm{V} 12}$ to our ectopic $\mathrm{H} 2 \mathrm{~B}$ cell lines described above without altering nuclear size (Supplementary Fig. 1c, d). Cells expressing ectopic wild-type $\mathrm{H} 2 \mathrm{~B}$ or $\mathrm{H} 2 \mathrm{~B}^{\mathrm{K} 15 \mathrm{R}}$ demonstrated a robust activation of SASP gene transcription in response to senescent cell-generated conditioned media (Fig. 5b). However, cells expressing $\mathrm{H} 2 \mathrm{~B}^{\mathrm{K} 20 \mathrm{R}}$ showed a dramatic and highly significant reduction in their ability to activate SASP gene transcription in response to senescent cellgenerated conditioned media. We confirmed by ChIP that macroH2A1 association with SASP genes assayed was significantly reduced specifically in $\mathrm{H}_{2} \mathrm{~B}^{\mathrm{K} 20 \mathrm{R}}$ expressing cells (Fig. 5c). Together, these results highlight the requirement for macroH2A1 to function in a specific chromatin context and demonstrate that the regulation of macroH2A1 transcriptional programs such as the SASP requires the local function of macroH $2 \mathrm{~A} 1$.

H2BK20ac is a beacon directing macroH2A1 deposition. $\mathrm{H} 2 \mathrm{~A}$ and $\mathrm{H} 2 \mathrm{~B}$-type histones are folded together as obligate dimers ${ }^{51}$.
Consequently, $\mathrm{H} 2 \mathrm{~A} / \mathrm{H} 2 \mathrm{~B}$ dimers are the physiological substrate for histone exchange machinery rather than monomers. Histones can be modified prior to deposition and certain modifications have been shown to be required for chromatin assembly ${ }^{52,53}$. We expressed $\mathrm{H} 2 \mathrm{~A}$ and $\mathrm{H} 2 \mathrm{~B}$ as a single-chain (one peptide) (Fig. 6a) using a similar strategy to one used in yeast ${ }^{54}$, to determine if $\mathrm{H} 2 \mathrm{BK} 20 \mathrm{ac}$ is required on the macroH2A1/H2B heterodimer or on the nucleosome-bound $\mathrm{H} 2 \mathrm{~B}$ as a genomic beacon. We generated single-chain constructs with just the HFD of macroH2A1 as we previously showed the macroH2A1 HFD was sufficient for accurate deposition (Fig. 1f) and were concerned the full length macroH2A1 protein would not fold correctly when fused to H2B. Wild-type $\mathrm{H} 2 \mathrm{~B}$ was also fused to canonical $\mathrm{H} 2 \mathrm{~A}$ as a negative control. We digested the chromatin fractions with MNase as shown previously for macroH2A1 ectopic mutants. A Flag immunoblot indicated the overwhelming majority of the ectopically expressed H2B-H2A-type single-chain proteins were predominantly chromatin incorporated (Fig. 6b).

Next, we determined the single-chain specification of deposition using ChIP-qPCR for Flag as well as endogenous macroH2A 1 as a positive control. There is a strong and highly significant correlation between deposition of endogenous macroH2A1 in the H2B-H2A-Flag single-chain expressing cells with the deposition of wild-type H2B-macroH2A1 $1^{1-122}$-Flag (Fig. 6c). Having established the wild-type H2BmacroH2A $1^{1-122}$-Flag protein behaves like endogenous macroH2A1, we next determined the role of the fused $\mathrm{H} 2 \mathrm{~B}$ on macroH2A1 deposition. As indicated in Fig. 6c, there is a strong 
a

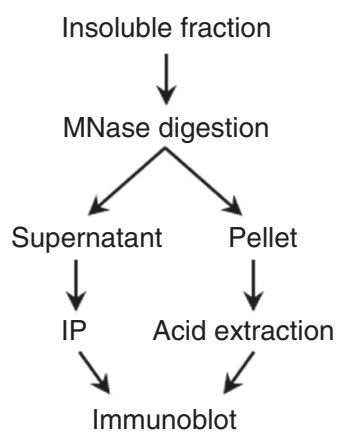

b

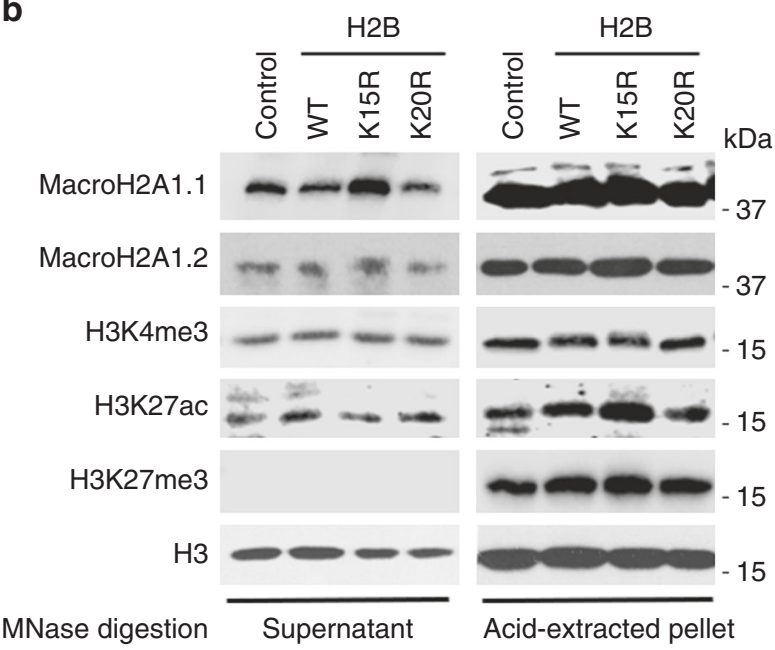

C

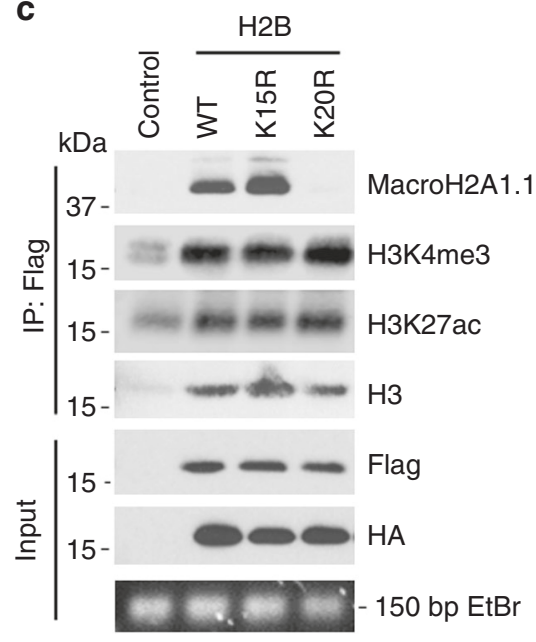

Fig. 4 MacroH2A1.1 associates with H2B K20 acetylation in mononucleosomes. a Schematic for micrococcal nuclease (MNase) digestion and fractionation of euchromatic mononucleosomes for immunoprecipitation (IP) using Flag antibody. b Immunoblots from fractionated MNase digested cells expressing H2B mutants described in (Fig. 3b) from the supernatant and acid-extracted pellet for indicated histones and histone PTMs. c Immunoblots for indicated histones and histone PTMs of mononucleosome Flag IP and input material from cells described in (Fig. 3b). Bottom box is ethidium bromide stained DNA band from MNase generated mononucleosomes. Three independent MNase digestions from distinct cell passages and Flag IPs demonstrated equivalent results

and significant correlation between the deposition of H2BmacroH2A $1^{1-122}$-Flag with constructs containing either the WT, K15R or K20R mutation on the fused H2B. Furthermore, there is no correlation between deposition of wild-type H2BmacroH2A1 ${ }^{1-122}$-Flag with H2B-H2A-Flag as expected from the uniform distribution of canonical $\mathrm{H} 2 \mathrm{~A}$ throughout the genome. Overall, this demonstrates that the H2B K20 in the targeted nucleosome, and not the $\mathrm{H} 2 \mathrm{~B}$ K20 in the macroH2A1/ $\mathrm{H} 2 \mathrm{~B}$ dimer, is required for macroH2Al deposition in $\mathrm{H} 2 \mathrm{Bac}$ chromatin.

\section{Discussion}

Our previous work demonstrated that macroH2A1 is found in two functionally distinct types of chromatin marked by either $\mathrm{H} 2 \mathrm{Bac}$ or by $\mathrm{H} 3 \mathrm{~K} 27 \mathrm{me} 3$ and that most macroH2A1-dependent transcriptional regulation occurs within its $\mathrm{H} 2 \mathrm{~B}$-acetylated subtype ${ }^{4,29}$. Here, we uncover the different rules governing macroH2A1 localization to these two types of chromatin, with multiple regions being sufficient for localization of macroH2A1 to H3K27me3-marked chromatin and the carboxyl-terminal half of the HFD being required for specification of macroH2A1containing H2B-acetylated chromatin. Previously, we demonstrated that macroH2A1.1 regulates the expression of its target genes through promotion of $\mathrm{H} 2 \mathrm{~B}$ acetylation at $\mathrm{K} 12$ and $\mathrm{K} 120^{4}$. Here, we show that macroH2A1.1 alone is not sufficient for regulation of these events and instead requires its localization to the appropriate chromatin environment to perform these functions. Importantly, we identify $\mathrm{H} 2 \mathrm{BK} 20 \mathrm{ac}$ as a critical PTM governing the localization and function of macroH2A1. Overall, our results demonstrate the critical role that localization of macroH2A1 plays in the regulation of its transcriptional programs, such as the SASP.

Our results demonstrate that multiple features of macroH2A1 are sufficient to target macroH2A 1 to its $\mathrm{H} 3 \mathrm{~K} 27 \mathrm{me} 3$-containing chromatin environment. This result is entirely consistent with previous studies that identified dispersed elements of macroH2A1 were each sufficient to target it to the $\mathrm{Xi}^{36,37}$. Xist noncoding RNA triggers the formation of the highly specific, dense Xi heterochromatin structure composed of partially redundant layers of repression, including $\mathrm{H} 3 \mathrm{~K} 27 \mathrm{me}$, DNA methylation, histone deacetylation and deposition of macroH2 $\mathrm{Al}^{55,56}$. Xist is required for the initiation of $\mathrm{X}$ chromosome inactivation, but it is not required for the maintenance of the Xi. However, Xist is required to maintain the association of macroH2A1 with the $\mathrm{Xi}^{57}$. Since the role of Xist is largely limited to triggering heterochromatin formation on the $\mathrm{X}$ chromosome, future studies are required to determine if an analogous mechanism exists to target macroH2A1 to autosomal H3K27me3-containing heterochromatic regions.

We show that criteria governing localization of macroH2A1 to $\mathrm{H} 2 \mathrm{Bac}$ chromatin are distinct from those governing localization to H3K27me3-containing chromatin (Fig. 7). Specifically, the carboxyl-terminal half of the HFD of macroH2A1 is uniquely required for accurate targeting to H2Bac-marked chromatin. And, while the beacon that recruits macroH2A1 to autosomal H3K27me3-containing chromatin is unknown, our results have determined that H2BK20ac targets macroH2A1 to its H2Bacetylated environment. H2BK20ac was recently shown to be highly associated with cell-type specific promoters and more predictive of functionally active enhancers than $\mathrm{H} 3 \mathrm{~K} 27 \mathrm{ac}^{58}$. H2BK20ac has also been shown to be highly correlated with H2BK120ac, especially at enhancers ${ }^{58}$. We previously determined that macroH2A1.1 regulates the transcription of its target genes in $\mathrm{H} 2 \mathrm{Bac}$ chromatin through PARP1-dependent recruitment of CBP, which acetylates $\mathrm{H} 2 \mathrm{~B}$ on $\mathrm{K} 12$ and $\mathrm{K} 120^{4,29}$, and now we demonstrate $\mathrm{H} 2 \mathrm{BK} 20 \mathrm{ac}$ targets its deposition to H2Bac chromatin. A key remaining goal is the identification of the upstream acetyltransferase responsible for $\mathrm{H} 2 \mathrm{BK} 20 \mathrm{ac}$ and subsequently the recruitment of macroH2A1 to H2B-acetylated chromatin.

With enhanced understanding of the key feature of macroH2A1 and the histone PTM required to specify macroH2A1-containing H2Bac chromatin, an important future goal is to identify the machinery that carries out this process. Multiple labs have performed various interaction studies to identify modulators of macroH2A1 function and deposition ${ }^{3,5,59}$. However, these studies have not identified a specific histone chaperone or ATP-dependent chromatin remodeling enzyme responsible for deposition of macroH2A. The exceptions come from the DNA damage field where macroH2A1 recruitment to sites of DNA damage have been 


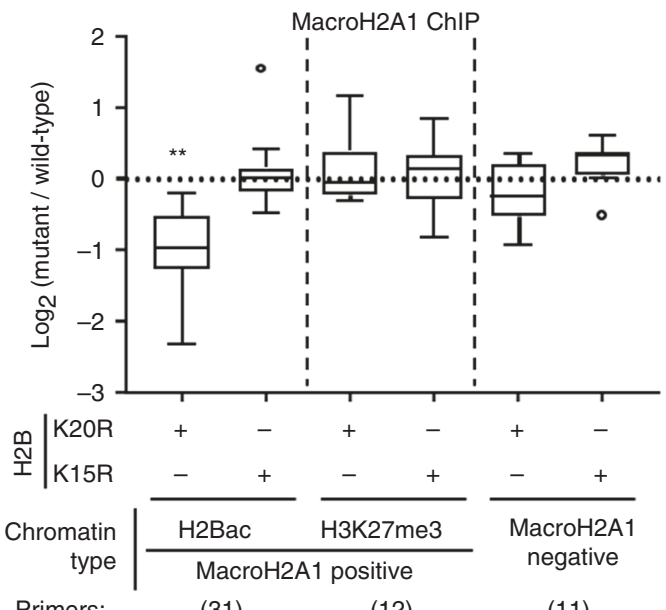

Primers: $\quad(31)$

(12)

$(11)$

b

$$
\text { qRT-PCR }
$$

C

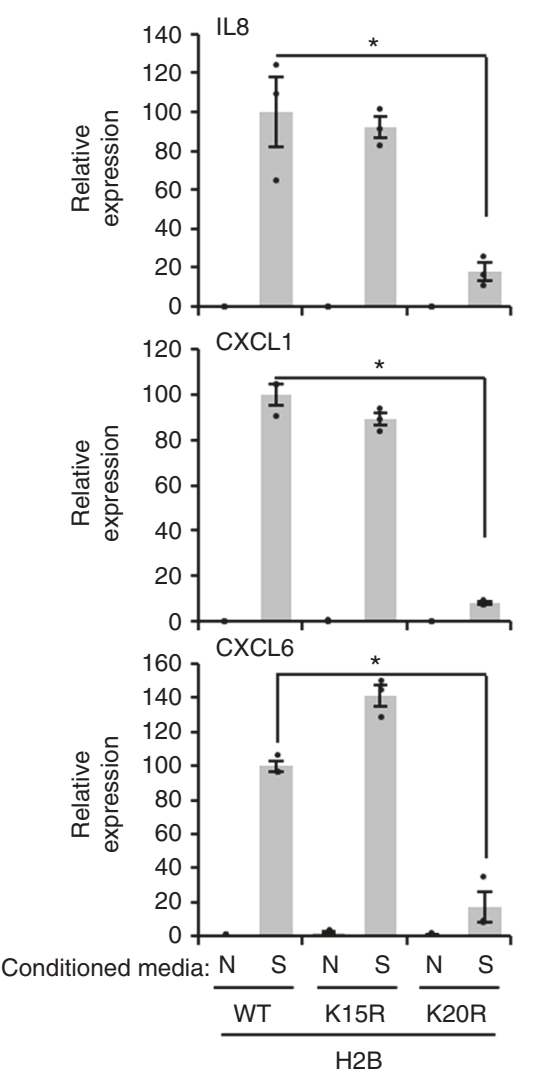

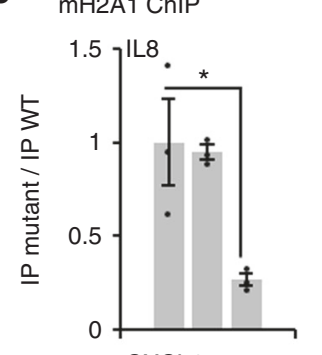
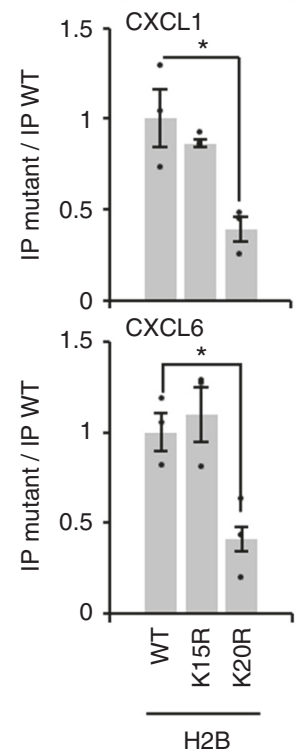

Fig. $5 \mathrm{H} 2 \mathrm{BK} 2 \mathrm{Oac}$ promotes macroH2A1 deposition into H2B-acetylated chromatin. a Endogenous macroH2A1 ChIP-qPCR from cells expressing $\mathrm{H} 2 \mathrm{~B}$ constructs described in Fig. $3 \mathrm{~b}$ using primers targeting locations within the indicated types of chromatin (regions containing either H3K27me3 or $\mathrm{H} 2 \mathrm{~B}$-acetylated chromatin that are associated with macroH2A1 or lack macroH2A1). Data is displayed as a ratio of mutant $\mathrm{H} 2 \mathrm{~B}$ to wild-type $\mathrm{H} 2 \mathrm{~B}$. Center line is the mean and bounds are $95 \%$ confidence interval of the mean. $\left({ }^{*} p=1.6 \times 10^{-11}\right)$. $\mathbf{b}$ RT-qPCR for the indicated SASP genes from cells described in Fig. $3 b$ that were subjected to paracrine senescence with conditioned media from $\mathrm{H}$-Ras ${ }^{\mathrm{V} 12}$-mediated oncogene induced senescent cells (S) or with cultured media from normal cells $(N)$ as a control. $\left({ }^{*} p<\right.$ 0.02). c Endogenous macroH2A1 ChIP-qPCR for SASP genes shown in $\mathbf{b}$ Bars represent the ratio of macroH2A1 association in mutant to wild-type H2B. $(p<0.03)$. Error bars, s.e.m., $n=3$ independent cell passages, $p$-values calculated from a two-tailed Student's t-test

reported by several groups ${ }^{12,35,60-62}$ where the histone chaperone APLF has been implicated and during replication stress where FACT has been implicated ${ }^{35}$. While the macroH2A1-deposition machinery has remained largely elusive, insights into factors that mediate the removal of macroH2A1 from chromatin have emerged. And ATRX, an ATP-dependent chromatin remodeler, has been implicated in this process ${ }^{59}$. In addition, our previous work demonstrated that the DNA-damage sensing kinase, ATM, promotes macroH2A1 removal specifically from $\mathrm{H} 2 \mathrm{Bac}$ chromatin during cellular senescence resulting in its global repositioning 29 . Our recent insights into the critical features governing macroH2A1 chromatin specification could lead to new assays to identify the machinery responsible for macroH2A deposition.

The carboxyl-terminal region of H2A.Z and H2A.X have previously been identified as critical for their genomic localization $^{42,63}$. Our results show that the carboxyl-terminal half of the HFD of macroH2A1, which harbors the docking domain, is critical for macroH2A1 localization to H2Bac chromatin. In H2A. $\mathrm{Z}$, the docking domain provides specificity for its deposition and functionality ${ }^{42}$. In macroH $2 \mathrm{~A} 1$, the main chain of the docking domain occupies the same path as canonical H2A, despite possessing regions of divergence (Supplementary Fig. 3) ${ }^{2}$. Our work suggests these changes confer specificity of macroH2A1 localization to $\mathrm{H} 2 \mathrm{Bac}$ chromatin. Binding to a chaperone can alter both the tertiary structure of the histone and the chaperone itself ${ }^{64}$. An additional carboxyl-terminal helix is formed in H2A.Z when bound by factors mediating its deposition, SWR1, ANP32E and $\mathrm{YL}^{44,51}$. This helical region, required for H2A.Z deposition, aligns to amino acids $95-103$ in macroH2A1, part of the region replaced in our mutants that are defective in localization to $\mathrm{H} 2 \mathrm{Bac}$ chromatin. Future experiments are required to determine if the deposition defective mutant is preventing a conformational change of the macroH $2 \mathrm{~A} 1 / \mathrm{H} 2 \mathrm{~B} /$ chaperone complex required for specific recognition and deposition into $\mathrm{H} 2 \mathrm{Bac}$ chromatin.

Trans-histone regulatory pathways, where modification of one histone in a nucleosome (e.g., H2B K120 ubiquitination) effects a second histone modification (e.g., H3 K79 methylation), are central features of chromatin regulatory mechanisms. Histone variants also participate in trans-histone regulatory processes ${ }^{65}$. For example, macroH2A1.1 promotes H2BK12ac and $\mathrm{H} 2 \mathrm{BK} 120 \mathrm{ac}^{4}$. Histone variants can also be affected by upstream histone PTMs. Case-in-point, H2A.Z is targeted to sites marked by certain histone acetylations (e.g., H4K16ac, and others) ${ }^{66-68}$. Here, we demonstrate that $\mathrm{H} 2 \mathrm{BK} 20 \mathrm{ac}$ is an upstream PTM regulating the localization of macroH2A1 to $\mathrm{H} 2 \mathrm{~B}$-acetylated chromatin. This result, together with the role macroH2A1 plays in promoting $\mathrm{H} 2 \mathrm{BK} 12 \mathrm{ac}$ and $\mathrm{H} 2 \mathrm{BK} 120 \mathrm{ac}$, firmly integrates macroH2A1 as an important hub in the broader histone code.

We have operated under a model in which macroH2A1 directly regulates transcription of its target genes by cofactor recruitment and modifying local chromatin structure. There is a variety of evidence that supports this model, most notably that macroH2A1 regulated genes are typically found in or near macroH2A1-containing chromatin ${ }^{4,21,29}$. However, a variety of other studies failed to identify a dominant role for macroH2A1 in the regulation of gene expression ${ }^{32,69,70}$. Recently, an indirect, global role for macroH2A1.1 in the regulation of mitochondrial function and cellular energy metabolism has been identified ${ }^{32}$. By capping PAR chains, macroH2A1.1 can inhibit PARP activity reducing nuclear $\mathrm{NAD}^{+}$flux and allowing a greater fraction of $\mathrm{NAD}^{+}$to be available for mitochondrial respiration. This effect is global, as it does not require macroH2A1.1 to directly regulate transcription. Interestingly, recent studies of macroH2A1 knockout mice have demonstrated phenotypes consistent with altered energy metabolism ${ }^{69,71}$. Overall, these findings require the field to carefully re-evaluate which functions of macroH2A1 play 
a

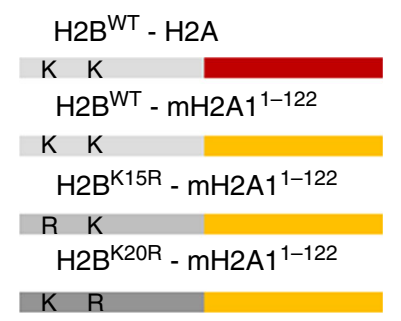

Residue: 1520

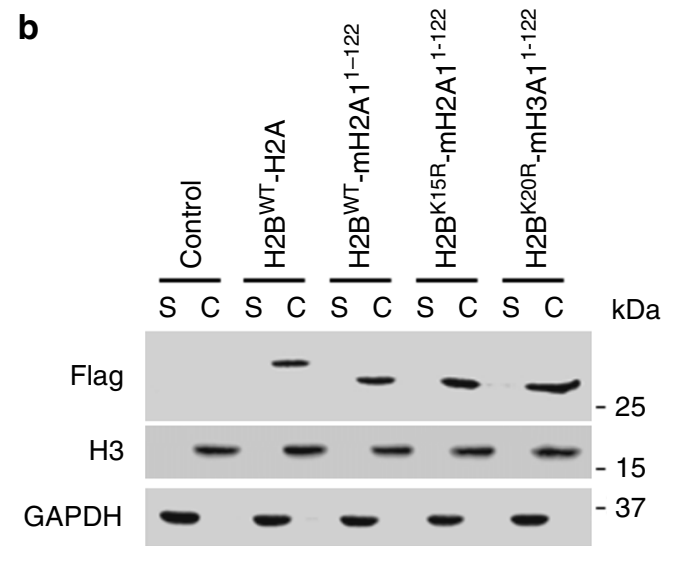

c

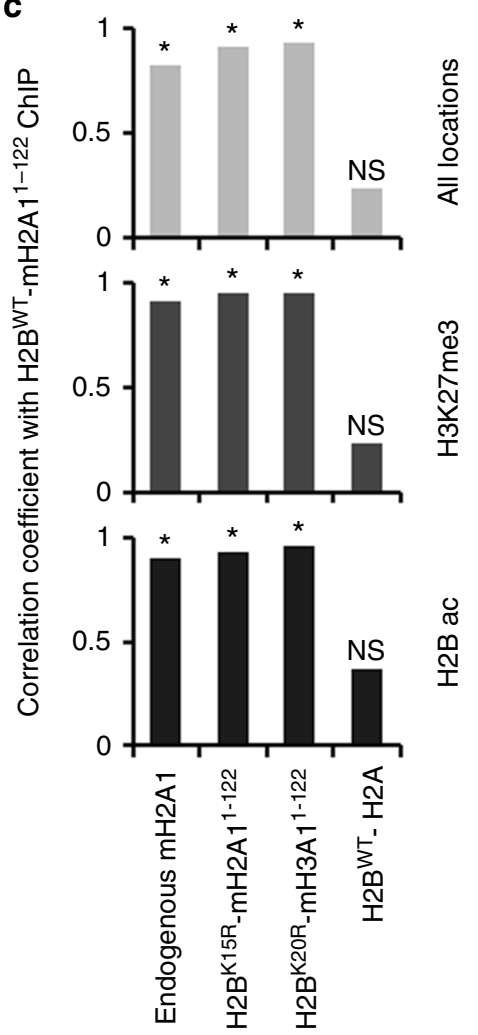

Fig. $6 \mathrm{H} 2 \mathrm{BK} 2 \mathrm{Oac}$ is a beacon targeting macroH2A1 deposition into $\mathrm{H} 2 \mathrm{~B}$-acetylated chromatin. a Schematic for single chain constructs with $\mathrm{H} 2 \mathrm{~B}$ fused directly to H2A-type histone representing obligate H2B-H2A dimers. b Immunoblots of soluble (S) and chromatin fractions (C) from IMR90 cells described in $\mathbf{a}$ for indicated proteins. Cells were fractionated with detergent. Insoluble fraction was digested to mononucleosomes with MNase to assess competence for chromatin incorporation. c Pearson's correlation coefficient calculated for Flag ChIP in $\mathrm{H}_{2} \mathrm{~B}^{\mathrm{W} T}$ - $\mathrm{mH} 2 \mathrm{~A} 1^{1-122}$-Flag expressing cells versus endogenous macroH2A1 ChIP in H2BWT-H2A-Flag expressing cells and versus Flag ChIP in single chain obligate histone dimer expressing cells described in a. $(n=3$ independent cell passages, ${ }^{\star} p<1 \times 10^{-10}$ from Pearson's product-moment correlation, $\mathrm{NS}=$ not significant)

a global role in cellular metabolism and which functions of macroH2A1 depend locally on its ability to regulate transcription. Here we demonstrate that the ability of macroH2A1 to promote $\mathrm{H} 2 \mathrm{BK} 12 \mathrm{ac}$ and $\mathrm{H} 2 \mathrm{BK} 120 \mathrm{ac}$ and regulate a key transcriptional program during senescence, the SASP, are local functions of macroH2A1 which require the carboxyl-terminal region of its HFD and H2BK20ac for its localization to target genes. These findings provide critical tools to definitively determine which of macroH2A1's biological functions are mediated by its global effect on energy metabolism and which are a local consequence of its role in transcriptional regulation.

\section{Methods}

Cell culture. IMR90 (CCL-186) (ATCC) primary human fetal lung fibroblast cells were immortalized using retroviral-mediated gene transfer of the hTERT gene (Addgene plasmid 1773) (IMR90-hTERT) ${ }^{14}$ and maintained in MEM supplemented with 10\% FBS. Human embryonic kidney 293T cells (HEK293T) (ATCC) were cultured in DMEM supplemented with 10\% FBS. Cell lines in culture were routinely tested for mycoplasma contamination MycoAlert ${ }^{\text {tx }}$ Mycoplasma Detection Kit (Lonza).

IMR90-hTERT cell lines expressing macroH2Al constructs were generated using the pLVX-Puro expression system (Clontech). IMR90-hTERT cell lines expressing H2B constructs were generated using pLVX-IRES-Puro and pLVXIRES-Neo vectors (Clontech). MacroH2A1 was cloned into and mutated within pLVX-Puro vector. H2B was cloned and mutated in pLVX-IRES-Puro and PLVXIRES-Neo. Single chain H2B-H2A-type construct were fused directly, minus the stop codon in $\mathrm{H} 2 \mathrm{~B}$ and the methionine in the $\mathrm{H} 2 \mathrm{~A}$-type histone. The $\mathrm{H} 2 \mathrm{~B}$ was expressed on the N-terminus and the H2A-type was on the C-terminus of the peptide. Lysine to arginine mutations were generated using standard Dpn1 mediated site-directed mutagenesis. The commercial $\mathrm{pBABE}$ and $\mathrm{pBABE}-\mathrm{H}-$ $\mathrm{Ras}^{\mathrm{V} 12}$ were gifts from William Hahn (Addgene plasmid \#9051). The retroviral or lentiviral infections were performed using X-tremeGENE ${ }^{\text {mi }} 9$ DNA Transfection Reagent (Roche) $)^{4}$. Three days post infection, the cells were selected for infection with $1 \mu \mathrm{g} / \mathrm{ml}$ puromycin and $/$ or $0.5 \mathrm{mg} / \mathrm{ml}$ neomycin.

Paracrine senescence was induced using conditioned media from $\mathrm{H}-\mathrm{Ras}^{\mathrm{V} 12}$ expressing senescent IMR90-hTERT cells (or normally growing IMR90-hTERT cells as a control) as previously described ${ }^{29}$. Briefly, conditioned media was collected from $\mathrm{H}$-Ras ${ }^{\mathrm{V} 12}$-induced senescent cells or normally growing cells as a control. Starting 14 days post-infection, the cultured medium was collected from the dish every 3 days, centrifuged at $3000 \mathrm{rpm}$ for $10 \mathrm{~min}$, then the supernatant was filtered through a $0.2 \mu \mathrm{m}$ pore filter (GE Healthcare). The resulting filtered medium was then mixed with an equal volume of MEM with $20 \%$ FBS and used as media for IMR90-hTERT cells expressing H2B constructs. Cells were harvested 3 days after treatment with conditioned media for RNA expression analysis.

Immunoblots and extraction of histones. For immunoblots, histones were either acid extracted ${ }^{4}$ or liberated by micrococcal nuclease digestion (MNase). Briefly, cells from a $10-\mathrm{cm}$ dish were lysed in $0.1 \%$ Triton X-100 lysis buffer $(10 \mathrm{mM}$ Tris, pH 7.9, 0.1\% Triton X-100, $100 \mathrm{mM} \mathrm{NaCl}, 1 \mathrm{mM}$ EDTA, 5\% glycerol, $1 \mathrm{mM}$ DTT and $1 \times$ protease-inhibitor cocktail (Roche, 11836170001)) and incubated with agitation at $4^{\circ} \mathrm{C}$ for $30 \mathrm{~min}$. Then, the lysate was centrifuged at $14,000 \mathrm{rpm}$ for 10 min at $4{ }^{\circ} \mathrm{C}$. The supernatant was collected as the soluble detergent lysate. For acid extraction, the insoluble pellets containing the histones were resuspended in $0.5 \mathrm{M}$ $\mathrm{HCl}$ at $4{ }^{\circ} \mathrm{C}$ for $2 \mathrm{~h}$ under constant agitation. The sample was spun at $14,000 \mathrm{rpm}$ for $10 \mathrm{~min}$ at $4{ }^{\circ} \mathrm{C}$. The resulting supernatant containing the histones was neutralized with $2 \mathrm{M}$ Tris base.

For MNase digestion, the insoluble pellet was gently resuspended in MNase digestion buffer (50 mM Tris 7.9, $25 \mathrm{mM} \mathrm{KCl}, 12.5 \%$ glycerol, $10 \mathrm{mM} \mathrm{CaCl}_{2}, 4 \mathrm{mM}$ $\mathrm{MgCl}_{2}$ ) and digested with MNase (NEB, M0247S) for $12 \mathrm{~min}$ at $37^{\circ} \mathrm{C}$. Digestion was stopped using MNase stop buffer (200 mM EDTA, $20 \mathrm{mM}$ Tris $7.9,0.1 \mathrm{mg} / \mathrm{ml}$ RNase). Ten microliters of the reaction was used to isolate DNA in order to confirm digestion to mononucleosomes. Briefly, the sample for DNA was cleared of protein with $0.4 \mathrm{mg} / \mathrm{ml}$ glycogen and proteinase $\mathrm{K}(2.5 \mathrm{U} / \mathrm{ml}$, Roche) in Txn stop buffer ( $20 \mathrm{mM}$ EDTA, $0.2 \mathrm{M} \mathrm{NaCl}$ and $1 \% \mathrm{SDS}$ ) at $37^{\circ} \mathrm{C}$ for $30 \mathrm{~min}$. The DNA was extracted with phenol/chloroform/isoamyl alcohol (25:24:1) and ethanol 


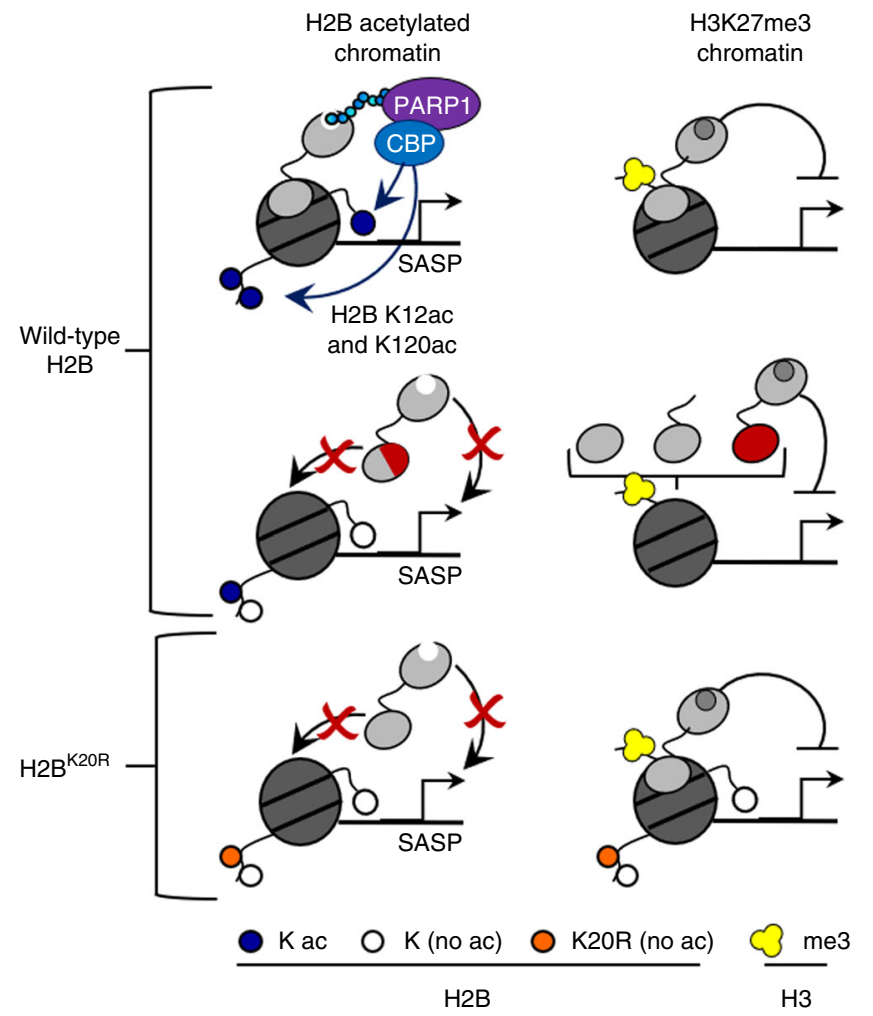

Fig. 7 Localization to the appropriate chromatin niche is required for local macroH2A1 function. There are different rules governing macroH2A1 localization to chromatin marked by either H2Bac or by H3K27me3. Multiple regions are sufficient for the localization of macroH2A1 to H3K27me3-marked chromatin. The carboxyl-terminal half of the HFD and $\mathrm{H} 2 \mathrm{BK} 2 \mathrm{Oac}$ are required for the specification of macroH2A1 to H2Bacetylated chromatin. MacroH2A1 must be localized to the appropriate chromatin environment, containing H2BK2Oac as a molecular beacon, to promote downstream acetylations and regulate transcription programs such as the SASP

precipitated. The resultant mononucleosome DNA was confirmed by running on $1.5 \%$ agarose gel.

The acid-extracted and MNase digested fractions were used for immunoblots of histones and the detergent lysates were used for immunoblots of all other factors. The detergent lysates, acid extracts, and MNase digested material were subjected to SDS-PAGE and immunoblotting (antibody information provided in Supplementary Table 2). HRP-conjugated goat anti-mouse or anti-rabbit secondary antibody was used for detection by ECL chemiluminescence according to the manufacturer's instructions (Thermo, Super Signal West Pico PLUS Chemiluminescent Substrate). All immunoblots have been repeated at least twice with independent biological samples. Uncropped immunoblots are provided in Supplementary Figure 7 .

Mononucleosome immunoprecipitation (MNase IP). Three $15-\mathrm{cm}$ dishes of IMR90-hTERT cells were grown to $95 \%$ confluence. The cells were collected, pelleted by centrifugation, combined by resuspending in $1 \mathrm{ml}$ of $0.1 \%$ Triton X-100 lysis buffer, and incubated at $4{ }^{\circ} \mathrm{C}$ for $30 \mathrm{~min}$ with agitation. The nuclei were collected by centrifugation at $4000 \mathrm{rpm}$ for $10 \mathrm{~min}$. The nuclear pellet was then gently resuspended in MNase buffer (three pellet volumes) and digested micrococcal nuclease $(5 \mu \mathrm{NEB}, \mathrm{M} 0247 \mathrm{~S})$ and digested at $37^{\circ} \mathrm{C}$ for $10 \mathrm{~min}$ with gentle vortexing every $2 \mathrm{~min}$. Digestion was stopped by adding MNase stop buffer ( 0.3 pellet volumes) and rocked at $4{ }^{\circ} \mathrm{C} 1.5 \mathrm{~h}$ to release mononucleosomes from euchromatin. The reaction was then spun at $13,000 \mathrm{rpm}$ for $10 \mathrm{~min}$. The supernatant contained the released mononucleosomes and the pellet had the nuclear mononucleosomes which were acid extracted as described above. Fifteen microliters of the supernatant of the MNase reaction was kept as input, and another $15 \mu \mathrm{l}$ was used to confirm digestion to mononucleosomes (as described above). Then remaining supernatant was combined with an equal volume of HEGTw/300 buffer (20 mM Tris, pH 7.9, $1 \mathrm{mM}$ EDTA, 5\% glycerol, 0.1\% Tween-20 and $300 \mathrm{mM}$ $\mathrm{NaCl}), 40 \mu \mathrm{l}$ protein $\mathrm{G}$ beads and either no antibody (negative control) or antibody against Flag. The reactions were rocked at $4{ }^{\circ} \mathrm{C}$ for $2 \mathrm{~h}$. The beads were spun at $4000 \mathrm{rpm}$ for $3 \mathrm{~min}$ and washed three times in HEGTw/300 buffer. The resulting beads were resuspended in 1X Laemli buffer, boiled, and centrifuged. The resulting supernatant containing eluted mononucleosome complexes were run on $10 \%$ or $20 \%$ SDS-polyacrylamide gels and immunoblotted for indicated proteins.

Chromatin immunoprecipitation (ChIP). Cells were grown to $90 \%$ confluence in $15-\mathrm{cm}$ dishes, cross-linked with $1 \%$ formaldehyde in PBS at room temperature for $10 \mathrm{~min}$ and then quenched in $125 \mathrm{mM}$ glycine for $5 \mathrm{~min}$. The cells were washed with cold PBS once and were collected by centrifugation and then sonicated in lysis buffer (50 mM Tris, pH 7.9, $10 \mathrm{mM}$ EDTA, $1 \%$ SDS, proteaseinhibitor cocktail and $1 \mathrm{mM}$ DTT) to generate chromatin fragments of $\sim 500 \mathrm{bp}$ in length. The material was clarified by $10 \mathrm{~min}$ centrifugation at $14,000 \mathrm{rpm}$ and $4{ }^{\circ} \mathrm{C}$, and $20 \mu \mathrm{l}$ supernatant was used as input for quantitation. The remaining supernatant was diluted ten-fold in dilution buffer $(20 \mathrm{mM}$ Tris, $\mathrm{pH} 7.9,2 \mathrm{mM}$ EDTA, $150 \mathrm{mM} \mathrm{NaCl}, 0.5 \%$ Triton X-100, protease-inhibitor cocktail (Roche, 11836170001 ) and $1 \mathrm{mM} \mathrm{DTT}$ ) and precleared with $20 \mu \mathrm{l}$ protein G-agarose beads at $4{ }^{\circ} \mathrm{C}$ for $2 \mathrm{~h}$. The supernatant was used in immunoprecipitations at $4{ }^{\circ} \mathrm{C}$ overnight with antibodies against macroH2A $1(8 \mu \mathrm{l})$, Flag $(5 \mu \mathrm{l})$ or histone $\mathrm{H} 3$ $(4 \mu \mathrm{l})$, as indicated (Supplementary Table 2) and were then incubated with $40 \mu \mathrm{l}$ protein G-agarose beads at $4^{\circ} \mathrm{C}$ for $2 \mathrm{~h}$. No-antibody (NA) controls were always included. The immunoprecipitated DNA was cleared of protein by digestion with $0.4 \mathrm{mg} / \mathrm{ml}$ glycogen and proteinase $\mathrm{K}(0.45 \mathrm{mg} / \mathrm{ml}$, Roche) in Txn stop buffer at $37^{\circ} \mathrm{C}$ for $1 \mathrm{~h}$. The DNA was then extracted with phenol/chloroform/ isoamyl alcohol (25:24:1) and ethanol precipitated. Quantitative real-time PCR with SYBR Green (Invitrogen) was used to determine enrichment of immunoprecipitated material relative to input with gene-specific primers to the specified regions (Supplementary Table 1).

ChIP-seq analyses. MacroH2A1 ChIP from GEO database (GSE54847) and sequencing reads for 26 additional histone marks in IMR90 cells were downloaded from the GEO website (GSE16256) and used to determine macroH2A1 enrichment. Enrichment analysis was performed as previously described ${ }^{4}$.

RNA purification and RT-qPCR. mRNA levels were analyzed by reverse transcription followed by quantitative PCR (RT-qPCR). RNA was isolated with TriPure (Roche) according to the manufacturer's protocol. The RNA was reverse transcribed with Moloney murine leukemia virus (MMLV) reverse transcriptase (Invitrogen) and a dT18 primer. cDNA, SYBR Green PCR master mix, and forward and reverse primers were used in 45 cycles of amplification $\left(95^{\circ} \mathrm{C}\right.$ for $15 \mathrm{~s}, 60^{\circ} \mathrm{C}$ for 1 minute) following 10 -min incubation at $95^{\circ} \mathrm{C}$, with a LightCycler 480 (Roche). The efficiency-corrected threshold cycle $(\Delta \mathrm{CT})$ method was used to determine the relative levels of RNA For transcription analysis, the expression was normalized to ACTB. Melting-curve analysis was performed to ensure specificity. Primer sequences are listed in Supplementary Table 3.

\section{Data availability}

The data that support the findings of this study are available from the corresponding author upon reasonable request.

Received: 29 December 2017 Accepted: 13 October 2018

Published online: 03 December 2018

\section{References}

1. Talbert, P. B. \& Henikoff, S. Histone variants--ancient wrap artists of the epigenome. Nat. Rev. Mol. Cell Biol. 11, 264-275 (2010).

2. Chakravarthy, S. et al. Structural characterization of the histone variant macroH2A. Mol. Cell. Biol. 25, 7616-7624 (2005).

3. Hussey, K. M. et al. The histone variant macroH2Al regulates target gene expression in part by recruiting the transcriptional coregulator PELP1. Mol. Cell. Biol. 34, 2437-2449 (2014).

4. Chen, H. et al. MacroH2A1.1 and PARP-1 cooperate to regulate transcription by promoting CBP-mediated H2B acetylation. Nat. Struct. Mol. Biol. 21, 981-989 (2014).

5. Ouararhni, K. et al. The histone variant mH2A1.1 interferes with transcription by down-regulating PARP-1 enzymatic activity. Genes Dev. 20, 3324-3336 (2006).

6. Dell'Orso, S. et al. The histone variant macroH2A1.2 is necessary for the activation of muscle enhancers and recruitment of the transcription factor Pbx1. Cell Rep. 14 (2016) https://doi.org/10.1016/j.celrep.2015.12.103

7. Hernández-Muñoz, I. et al. Stable X chromosome inactivation involves the PRC1 Polycomb complex and requires histone MACROH2A1 and the CULLIN3/SPOP ubiquitin E3 ligase. Proc. Natl Acad. Sci. USA 102, 7635-7640 (2005). 
8. Kim, J. et al. Regulation of breast cancer-induced osteoclastogenesis by MacroH2A1.2 involving EZH2-mediated H3K27me3. Cell Rep. 24, 224-237 (2018).

9. Kim, J.-M. et al. MacroH2A1.2 inhibits prostate cancer-induced osteoclastogenesis through cooperation with HP1a and H1.2. Oncogene 1 (2018). https://doi.org/10.1038/s41388-018-0356-3

10. Gamble, M. J., Frizzell, K. M., Yang, C., Krishnakumar, R. \& Kraus, W. L. The histone variant macroH2A1 marks repressed autosomal chromatin, but protects a subset of its target genes from silencing. Genes Dev. 24, 21-32 (2010).

11. Kustatscher, G., Hothorn, M., Pugieux, C., Scheffzek, K. \& Ladurner, A. G. Splicing regulates NAD metabolite binding to histone macroH2A. Nat. Struct. Mol. Biol. 12, 624-625 (2005).

12. Timinszky, G. et al. A macrodomain-containing histone rearranges chromatin upon sensing PARP1 activation. Nat. Struct. Mol. Biol. 16, 923-929 (2009).

13. Costanzi, C. \& Pehrson, J. R. MACROH2A2, a new member of the MARCOH2A core histone family. J. Biol. Chem. 276, 21776-21784 (2001).

14. Novikov, L. et al. QKI-mediated alternative splicing of the histone variant MacroH2A1 regulates cancer cell proliferation. Mol. Cell. Biol. 31, 4244-4255 (2011).

15. Sporn, J. C. et al. Histone macroH2A isoforms predict the risk of lung cancer recurrence. Oncogene 28, 3423-3428 (2009).

16. Sporn, J. C. \& Jung, B. Differential regulation and predictive potential of macroH2A1 isoforms in colon cancer. Am. J. Pathol. 180, 2516-2526 (2012).

17. Kim, J. M., Heo, K., Choi, J., Kim, K. \& An, W. The histone variant MacroH2A regulates $\mathrm{Ca}^{+}+$influx through TRPC3 and TRPC6 channels. Oncogenesis 2, e77 (2013).

18. Kapoor, A. et al. The histone variant macroH2A suppresses melanoma progression through regulation of CDK8. Nature 468, 1105-1109 (2010).

19. Dardenne, E. et al. Splicing switch of an epigenetic regulator by RNA helicases promotes tumor-cell invasiveness. Nat. Struct. Mol. Biol. 19, 1139-1146 (2012).

20. Ladurner, A. G. Inactivating chromosomes: a macro domain that minimizes transcription. Mol. Cell 12, 1-2 (2003).

21. Buschbeck, M. et al. The histone variant macroH2A is an epigenetic regulator of key developmental genes. Nat. Struct. Mol. Biol. 16, 1074-1079 (2009).

22. Changolkar, L. N. et al. Developmental changes in histone macroH2A1mediated Gene regulation. Mol. Cell. Biol. 27, 2758-2764 (2007).

23. Pasque, V. et al. Histone variant macroH2A marks embryonic differentiation in vivo and acts as an epigenetic barrier to induced pluripotency. J. Cell. Sci. 125, 6094-6104 (2012).

24. Barrero, M. J. et al. Macrohistone variants preserve cell Identity by preventing the gain of H3K4me2 during reprogramming to pluripotency. Cell Rep. 1-7 (2013). https://doi.org/10.1016/j.celrep.2013.02.029

25. Gaspar-Maia, A. et al. MacroH2A histone variants act as a barrier upon reprogramming towards pluripotency. Nat. Commun. 4, 1565 (2013).

26. Pehrson, J. R., Costanzi, C. \& Dharia, C. Developmental and tissue expression patterns of histone macroH2A1 subtypes. J. Cell. Biochem. 65, 107-113 (1997).

27. Wan, D. et al. MacroH2A1.1 cooperates with EZH2 to promote adipogenesis by regulating Wnt signaling. J. Mol. Cell Biol. 9, 325-337 (2017).

28. Buschbeck, M. \& Hake, S. B. Variants of core histones and their roles in cell fate decisions, development and cancer. Nat. Rev. Mol. Cell Biol. 18, 299-314 (2017).

29. Chen, $\mathrm{H}$. et al. MacroH2A1 and ATM Play opposing roles in paracrine senescence and the senescence-associated secretory phenotype. Mol. Cell 59, 719-731 (2015).

30. Zhang, R. et al. Formation of macroH2A-containing senescence-associated heterochromatin foci and senescence driven by ASF1a and HIRA. Dev. Cell 8, 19-30 (2005).

31. Coppé, J.-P., Desprez, P.-Y., Krtolica, A. \& Campisi, J. The senescenceassociated secretory phenotype: the dark side of tumor suppression. Annu. Rev. Pathol. 5, 99-118 (2010).

32. Marjanović, M. P. et al. MacroH2A1.1 regulates mitochondrial respiration by limiting nuclear $\mathrm{NAD}^{+}$consumption. Nat. Publ. Gr. (2017) https://doi.org/ $10.1038 / \mathrm{nsmb} .3481$

33. Changolkar, L. N. et al. Genome-wide distribution of macroH2Al histone variants in mouse liver chromatin. Mol. Cell. Biol. 30, 5473-5483 (2010).

34. Creppe, C. et al. MacroH2A1 regulates the balance between self-renewal and differentiation commitment in embryonic and adult stem cells. Mol. Cell. Biol. 32, 1442-1452 (2012).

35. Kim, J. et al. Replication stress shapes a protective chromatin environment across Fragile genomic regions. Mol. Cell 69, 1-12 (2017).

36. Nusinow, D. A. et al. The histone domain of macroH2A1 contains several dispersed elements that are each sufficient to direct enrichment on the inactive X chromosome. J. Mol. Biol. 371, 11-18 (2007).

37. Chadwick, B. P., Valley, C. M. \& Willard, H. F. Histone variant macroH2A contains two distinct macrochromatin domains capable of directing
macroH2A to the inactive X chromosome. Nucleic Acids Res. 29, 2699-2705 (2001).

38. Bernstein, B. E. et al. The NIH Roadmap Epigenomics Mapping Consortium. Nat. Biotechnol. 28, 1045-1048 (2010).

39. Pehrson, J. R. \& Fuji, R. N. Evolutionary conservation of histone macroH2A subtypes and domains. Nucleic Acids Res. 26, 2837-2842 (1998).

40. Chakravarthy, S. \& Luger, K. The histone variant macro-H2A preferentially forms 'hybrid nucleosomes'. J. Biol. Chem. 281, 25522-25531 (2006).

41. Richmond, R. K., Sargent, D. F., Richmond, T. J., Luger, K. \& Ma, A. W. Crystal structure of the nucleosome core particle at $2.8 \mathrm{~A}^{\circ}$ resolution. Nature 7, 251-260 (1997).

42. Wang, A. Y., Aristizabal, M. J., Ryan, C., Krogan, N. J. \& Kobor, M. S. Key functional regions in the histone variant H2A.Z C-terminal docking domain Mol. Cell. Biol. 31, 3871-3884 (2011).

43. Jensen, K., Santisteban, M. S., Urekar, C. \& Smith, M. M. Histone H2A.Z acid patch residues required for deposition and function. Mol. Genet. Genom. 285, 287-296 (2011)

44. Wu, W.-H. et al. Swc2 is a widely conserved H2AZ-binding module essential for ATP-dependent histone exchange. Nat. Struct. Mol. Biol. 12, 1064-1071 (2005).

45. Abell, A. N. et al. MAP3K4/CBP-regulated H2B acetylation controls epithelial-mesenchymal transition in trophoblast stem cells. Cell Stem Cell 8, 525-537 (2011).

46. Boija, A. et al. CBP regulates recruitment and release of promoter-proximal RNA Polymerase II. Mol. Cell 68, 491-503 (2017). e5.

47. Dancy, B. M. \& Cole, P. A. Protein lysine acetylation by p300/CBP. Chem. Rev. 115, 2419-2452 (2015).

48. Mieczkowski, J. et al. MNase titration reveals differences between nucleosome occupancy and chromatin accessibility. Nat. Commun. 7, 11485 (2016).

49. Krtolica, A., Parrinello, S., Lockett, S., Desprez, P.-Y. \& Campisi, J. Senescent fibroblasts promote epithelial cell growth and tumorigenesis: a link between cancer and aging. Proc. Natl Acad. Sci. USA 98, 12072-12077 (2001).

50. Kuilman, T. et al. Oncogene-Induced Senescence relayed by an interleukindependent inflammatory network. Cell 133, 1019-1031 (2008).

51. Mizuguchi, G. et al. ATP-driven exchange of histone $\mathrm{H} 2 \mathrm{AZ}$ variant catalyzed by SWR1 chromatin remodeling complex. Science 303, 343-348 (2004).

52. Wang, W. L. et al. Phosphorylation and arginine methylation mark histone H2A prior to deposition during Xenopus laevis development. Epigenetics Chromatin 7, 22 (2014).

53. Loyola, A., Bonaldi, T., Roche, D., Imhof, A. \& Almouzni, G. PTMs on H3 variants before chromatin assembly potentiate their final epigenetic state. Mol. Cell 24, 309-316 (2006).

54. Nakabayashi, Y., Kawashima, S., Enomoto, T., Seki, M. \& Horikoshi, M. Roles of common subunits within distinct multisubunit complexes. Proc. Natl Acad. Sci. USA 111, 699-704 (2014).

55. Penny, G. D., Kay, G. F., Sheardown, S. A., Rastan, S. \& Brockdorff, N. Requirement for Xist in X chromosome inactivation. Nature 379, 131-137 (1996).

56. Csankovszki, G., Nagy, A. \& Jaenisch, R. Synergism of Xist RNA, DNA methylation, and histone hypoacetylation in maintaining $\mathrm{X}$ chromosome inactivation. J. Cell. Biol. 153, 773-783 (2001).

57. Csankovszki, G., Panning, B., Bates, B., Pehrson, J. R. \& Jaenisch, R. Conditional deletion of Xist disrupts histone macroH2A localization but not maintenance of X inactivation. Nat. Genet. 22, 323-324 (1999).

58. Kumar, V. et al. Comprehensive benchmarking reveals H2BK20 acetylation as a distinctive signature of cell-state-specific enhancers and promoters. Genome Res. 26, 612-623 (2016).

59. Ratnakumar, K. et al. ATRX-mediated chromatin association of histone variant macroH2A1 regulates a-globin expression. Genes Dev. 26, 433-438 (2012).

60. Xu, C., Xu, Y., Gursoy-Yuzugullu, O. \& Price, B. D. The histone variant macroH2A1.1 is recruited to DSBs through a mechanism involving PARP1. FEBS Lett. 586, 3920-3925 (2012).

61. Mehrotra, P. V. et al. DNA repair factor APLF is a histone chaperone. Mol. Cell 41, 46-55 (2011).

62. Khurana, S. et al. A macrohistone variant links dynamic chromatin compaction to BRCA1-dependent genome maintenance. Cell Rep. 8, 1049-1062 (2014).

63. Latrick, C. M. et al. Molecular basis and specificity of H2A.Z-H2B recognition and deposition by the histone chaperone YL1. Nat. Struct. Mol. Biol. 23, 309-316 (2016).

64. Warren, C. \& Shechter, D. Fly fishing for histones: catch and release by histone chaperone intrinsically disordered regions and acidic stretches. J. Mol. Biol. 429, 2401-2426 (2017)

65. Lee, J. S., Smith, E. \& Shilatifard, A. The language of histone crosstalk. Cell 142, 682-685 (2010). 
66. Altaf, M. et al. NuA4-dependent acetylation of nucleosomal histones $\mathrm{H} 4$ and $\mathrm{H} 2 \mathrm{~A}$ directly stimulates incorporation of H2A.Z by the SWR1 complex. J. Biol. Chem. 285, 15966-15977 (2010).

67. Wan, Y. et al. Role of the histone variant H2A.Z/Htzlp in TBP recruitment, chromatin dynamics, and regulated expression of oleate-responsive genes. Mol. Cell. Biol. 29, 2346-2358 (2009).

68. Shia, W.-J., Li, B. \& Workman, J. L. SAS-mediated acetylation of histone H4 Lys 16 is required for H2A.Z incorporation at subtelomeric regions in Saccharomyces cerevisiae. Genes Dev. 20, 2507-2512 (2006).

69. Sheedfar, F. et al. Genetic ablation of macrohistone H2A1 leads to increased leanness, glucose tolerance and energy expenditure in mice fed a high-fat diet. Int. J. Obes. 39, 331-338 (2015).

70. Posavec, M., Timinszky, G. \& Buschbeck, M. Macro domains as metabolite sensors on chromatin. Cell. Mol. Life Sci. 70, 1509-1524 (2013).

71. Pehrson, J. R., Changolkar, L. N., Costanzi, C. \& Leu, N. A. Mice without macroH2A histone variants. Mol. Cell. Biol. 34, 4523-4533 (2014).

\section{Acknowledgements}

This work was supported by the NIH training grant T32GM007491-38 (P.D.R.), grant R01CA155232 (M.J.G.), and P30CA013330 (Einstein Cancer Center Grant).

\section{Author contributions}

Experiments were conducted by P.D.R. P.D.R. designed and performed experiments, analyzed the data and wrote the manuscript. M.J.G. designed experiments and wrote the manuscript. Manuscript was prepared by P.D.R. and edited by M.J.G.

\section{Additional information}

Supplementary Information accompanies this paper at https://doi.org/10.1038/s41467018-07189-8.

Competing interests: The authors declare no competing interests.

Reprints and permission information is available online at http://npg.nature.com/ reprintsandpermissions/

Publisher's note: Springer Nature remains neutral with regard to jurisdictional claims in published maps and institutional affiliations.

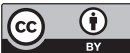

Open Access This article is licensed under a Creative Commons Attribution 4.0 International License, which permits use, sharing, adaptation, distribution and reproduction in any medium or format, as long as you give appropriate credit to the original author(s) and the source, provide a link to the Creative Commons license, and indicate if changes were made. The images or other third party material in this article are included in the article's Creative Commons license, unless indicated otherwise in a credit line to the material. If material is not included in the article's Creative Commons license and your intended use is not permitted by statutory regulation or exceeds the permitted use, you will need to obtain permission directly from the copyright holder. To view a copy of this license, visit http://creativecommons.org/ licenses/by/4.0/.

(C) The Author(s) 2018 